Pacific Journal of Mathematics

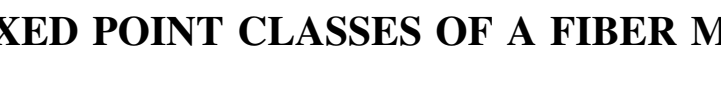




\section{FIXED POINT CLASSES OF A FIBER MAP}

\section{Cheng-Ye You}

Let $(E, p, B)$ be a fiber space with $E, B$ and all fibers compact connected ANR's. Let $f: E \rightarrow E$ be a fiber map, then $f$ induces $\bar{f}: B \rightarrow B$. For each fixed point $b$ of $\bar{f}$, we define $f_{b}=f \mid p^{-1}(b): p^{-1}(b) \rightarrow p^{-1}(b)$. Then $p \circ f=\bar{f} \circ p$ and $i_{b} \circ f_{b}=f \circ i_{b}$, where $i_{b}$ is the inclusion map. We have Nielsen numbers $N(f), N(\bar{f})$ and $N\left(f_{b}\right)$. A product formula relating these Nielsen numbers was published by Brown in 1967. There have been several improvements of the formula since that time.

In this paper, we study the structure of the fixed point classes of $f$, and prove some theorems about the product formula of the Nielsen number of a fiber map, which imply results of Fadell and of Pak.

Throughout this paper we assume all spaces are path-connected and all fiber spaces are Hurewicz fiber spaces.

I am grateful to Professor Boju Jiang (Po-chu Chiang) for some important suggestions.

I would also like to thank the referee for his help.

1. Fixed point $H$-classes. The concept of fixed point $H$-classes is presented in [8] and [7]. For convenience of calculation, we give its definition a precise algebraic formulation.

Let $X$ be a space, and $H$ be a normal subgroup of $\pi_{1}(X)$ (which means that for each $x \in X$, a normal subgroup $H(x)$ of $\pi_{1}(X, x)$ is defined, such that for any path $w$ in $X$ from $x$ to $x^{\prime}$, we have $w_{*}(H(x))=H\left(x^{\prime}\right)$, where $w_{*}: \pi_{1}(X, x) \rightarrow \pi_{1}\left(X, x^{\prime}\right)$ is defined by $w_{*}(\langle a\rangle)=$ $\left\langle w^{-1} a w\right\rangle$, for any $\left.\langle a\rangle \in \pi_{1}(X, x)\right)$. Two paths $c, d$ in $X$ are said to be $H$-homotopic and written $c \stackrel{\boldsymbol{H}}{\simeq} d$, if $c(0)=d(0), c(1)=d(1)$ and $\left\langle c d^{-1}\right\rangle \epsilon$ $H$. One can easily see that when $c \stackrel{\boldsymbol{H}}{\simeq} d$, then $d \stackrel{\boldsymbol{H}}{\simeq} c, c^{-1} \stackrel{\boldsymbol{H}}{\simeq} d^{-1}$ and also $u c \stackrel{\boldsymbol{H}}{\simeq} u d, c v \stackrel{\boldsymbol{H}}{\simeq} d v$ if $u c$ and $c v$ are well-defined.

Let $x \in X$, we can think of every element of $\pi_{1}(X, x) / H(x)$ as $\mathrm{a} \stackrel{\boldsymbol{H}}{\simeq}$ equivalence class of loops based at $x$. Let $\langle a\rangle_{H}$ denote the $\stackrel{\boldsymbol{H}}{\simeq}$ equivalence class of the loop $a$. For each path $w$ in $X$ from $x$ to $x^{\prime}$, let $w_{H}: \pi_{1}(X, x) / H(x) \rightarrow \pi_{1}\left(X, x^{\prime}\right) / H\left(x^{\prime}\right)$ be the homomorphism induced by $w_{*}$, that is, $w_{H}\left(\langle a\rangle_{H}\right)=\left\langle w^{-1} a w\right\rangle_{H}$.

Suppose that a map $f: X \rightarrow X$ satisfies $f_{\pi}(H) \subset H$ (it means that for any $x \in X, f_{\pi}(H(x)) \subset H(f(x))$ where $f_{\pi}: \pi_{1}(X, x) \rightarrow \pi_{1}(X, f(x))$ is the induced homomorphism). Then, for each $x \in X, f_{\pi}: \pi_{1}(X, x) \rightarrow$ $\pi_{1}(X, f(x))$ induces a homomorphism $f_{H}: \pi_{1}(X, x) / H(x) \rightarrow \pi_{1}(X, f(x)) /$ 


\section{$H(f(x))$.}

Let $\Phi(f)$ be the set of fixed points of $f$. Any two fixed points $x, x^{\prime} \in \Phi(f)$ are said to be in the same H-class, if there is a path $c$ in $X$ from $x$ to $x^{\prime}$ such that $c \stackrel{H}{\simeq} f \circ c$. Then $\Phi(f)$ is divided into a finite number of equivalence classes, called nonempty fixed point $H$-classes of $f$. Let $\Phi_{H}^{\prime}(f)$ denote the set of nonempty fixed point $H$-classes of $f$.

Let $x_{0} \in X$ and let $w$ be a path in $X$ from $x_{0}$ to $f\left(x_{0}\right)$. We define a homomorphism $\tilde{f}_{H}=w_{H}^{-1} \circ f_{H}: \pi_{1}\left(X, x_{0}\right) / H\left(x_{0}\right) \rightarrow \pi_{1}\left(X, x_{0}\right) / H\left(x_{0}\right)$, so $\widetilde{f}_{H}\left(\langle a\rangle_{H}\right)=\left\langle w(f \circ a) w^{-1}\right\rangle_{H}$. Any two elements $\alpha, \alpha^{\prime}$ of $\pi_{1}\left(X, x_{0}\right) / H\left(x_{0}\right)$ are said to be " $\sim$ equivalent" (written $\alpha \sim \alpha^{\prime}$ ) if there is a $\gamma \in$ $\pi_{1}\left(X, x_{0}\right) / H\left(x_{0}\right)$ such that $\alpha^{\prime}=\gamma \alpha \widetilde{f}_{H}\left(\gamma^{-1}\right)$. Let $\nabla_{H}\left(f ; x_{0}, w\right)$ denote the set of $\sim$ equivalence classes, and let $[\alpha]$ denote the $\sim$ equivalence class of $\alpha$.

LEMMA 1.1. For each $x \in \Phi(f)$, the set $\left\{\left\langle c\left(f \circ c^{-1}\right) w^{-1}\right\rangle_{H} \mid\right.$ for any path $c$ from $x_{0}$ to $\left.x\right\}$ is exactly a equivalence class, so $x$ determines an element of $\nabla_{H}\left(f ; x_{0}, w\right)$. Two fixed points determine the same element of $\nabla_{H}\left(f ; x_{0}, w\right)$ if and only if they are in the same $H$-class.

Proof. We take a path $c_{0}$ from $x_{0}$ to $x$. For any path $c$ from $x_{0}$ to $x, c c_{0}^{-1}$ is a loop based at $x_{0}$. Then

$$
\begin{aligned}
\left\langle c(f \circ c)^{-1} w^{-1}\right\rangle_{H} & =\left\langle c c_{0}^{-1} c_{0}\left(f \circ c_{0}^{-1}\right)\left(f \circ c_{0} c^{-1}\right) w^{-1}\right\rangle_{H} \\
& =\left\langle c c_{0}^{-1}\right\rangle_{H}\left\langle c_{0}\left(f \circ c_{0}^{-1}\right) w^{-1}\right\rangle_{H} \tilde{f}_{H}\left(\left\langle c c_{0}^{-1}\right\rangle_{H}\right) \\
& \sim\left\langle c_{0}\left(f \circ c_{0}^{-1}\right) w^{-1}\right\rangle_{H} .
\end{aligned}
$$

Hence $\left\{\left\langle c\left(f \circ c^{-1}\right) w^{-1}\right\rangle_{H} \mid\right.$ for any path $c$ from $x_{0}$ to $\left.x\right\} \subset\left[\left\langle c_{0}\left(f \circ c_{0}^{-1}\right) w^{-1}\right\rangle_{H}\right]$.

On the other hand, if $\gamma=\langle r\rangle_{H}$, then (writing $c=r c_{0}$ )

$$
\left\langle c\left(f \circ c^{-1}\right) w^{-1}\right\rangle_{H}=\gamma\left\langle c_{n}\left(f \circ c_{0}^{-1}\right) w^{-1}\right\rangle_{H} \tilde{f}_{H}\left(\gamma^{-1}\right) .
$$

Hence $\left[\left\langle c_{0}\left(f \circ c_{0}^{-1}\right) w^{-1}\right\rangle_{H}\right] \subset\left\{\left\langle c\left(f \circ c^{-1}\right) w^{-1}\right\rangle_{H} \mid\right.$ for any path $c$ from $x_{0}$ to $x\}$. Thus we get the first conclusion.

Let $x, x^{\prime} \in \Phi(f)$ be in the same $H$-class. Take a path $d$ from $x$ to $x^{\prime}$ such that $d \stackrel{\boldsymbol{H}}{\simeq}(f \circ d)$, and a path $c$ from $x_{0}$ to $x$. Then $c d$ is a path from $x_{0}$ to $x^{\prime}$, and

$$
\left\langle c d\left(f \circ(c d)^{-1}\right) w^{-1}\right\rangle_{H}=\left\langle c d\left(f \circ d^{-1}\right)\left(f \circ c^{-1}\right) w^{-1}\right\rangle_{H}=\left\langle c\left(f \circ c^{-1}\right) w^{-1}\right\rangle_{H} .
$$

Hence $x, x^{\prime}$ determine the same element $\left[\left\langle c\left(f \circ c^{-1}\right) w^{-1}\right\rangle_{H}\right] \in \nabla_{H}\left(f ; x_{0}, w\right)$.

Conversely, if $x, x^{\prime} \in \Phi(f)$ determine the same element, then, according to the first conclusion, we can find paths $c, c^{\prime}$ from $x_{0}$ to $x, x^{\prime}$ respectively such that

$$
c\left(f \circ c^{-1}\right) w^{-1} \stackrel{\boldsymbol{H}}{\simeq} c^{\prime}\left(f \circ c^{\prime-1}\right) w^{-1} .
$$


It follows that $c^{-1} c^{\prime} \stackrel{\boldsymbol{H}}{\simeq} f \circ\left(c^{-1} c^{\prime}\right)$. Since $c^{-1} c^{\prime}$ is a path from $x$ to $x^{\prime}$, we get that $x, x^{\prime}$ are in the same $H$-class.

Lemma 1.1 permits us to define an injection $\rho\left(x_{0}, w\right): \Phi_{I I}^{\prime}(f) \rightarrow$ $\nabla_{H}\left(f ; x_{0}, w\right)$ by $\rho\left(x_{0}, w\right)(\boldsymbol{F})=\left[\left\langle c\left(f \circ c^{-1}\right) w^{-1}\right\rangle_{H}\right]$, where $x \in \boldsymbol{F} \in \Phi_{H}^{\prime}(f)$ and $c$ is a path from $x_{0}$ to $x$.

The set $\nabla_{H}\left(f ; x_{0}, w\right)$ depends on the pair $\left(x_{0}, w\right)$. Let $(x, w)$ and $\left(x^{\prime}, w^{\prime}\right)$ be two such pairs. If $x=x^{\prime}, w \stackrel{\boldsymbol{H}}{\simeq} w^{\prime}$, then $w_{H I}=w_{I I}^{\prime}$ and $\widetilde{f}_{H}, \sim$ equivalence are the same, so $\nabla_{H}(f ; x, w)=\nabla_{H}\left(f^{\prime} ; x^{\prime}, w^{\prime}\right)$. In general, however, $\nabla_{H}(f ; x, w)$ is different from $\nabla_{H}\left(f ; x^{\prime}, w^{\prime}\right)$. We now show the relation between them. It is easy to prove that:

LEMMA 1.2. (a) If $\langle a\rangle_{H},\left\langle a^{\prime}\right\rangle_{I I} \in \pi_{1}(X, x) / H(x)$ are $\sim$ equivalent and $u$ is a path from $x$ to $x^{\prime}$, then

$$
\left\langle u^{-1} a w(f \circ u) w^{\prime-1}\right\rangle_{I I} \sim\left\langle u^{-1} a^{\prime} w(f \circ u) w^{\prime-1}\right\rangle_{I I} .
$$

Thus we can define a transformation $\nu: \nabla_{H}(f ; x, w) \rightarrow \nabla_{H}\left(f ; x^{\prime}, w^{\prime}\right)$ by

$$
\nu\left(\left[\langle a\rangle_{I I}\right]\right)=\left[\left\langle u^{-1} a w(f \circ u) w^{\prime-1}\right\rangle_{H}\right] .
$$

(b) $\nu$ is independent of the choice of path $u$.

(c) When $x=x^{\prime}$ and $w \stackrel{\boldsymbol{H}}{\simeq} w^{\prime}$, then $\nu$ is the identity.

(d) If we have another pair $\left(x^{\prime \prime}, w^{\prime \prime}\right)$, then the diagram

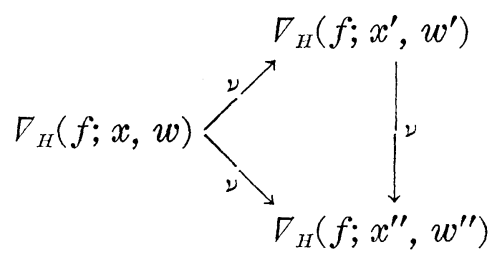

is commutative.

(e) We have a commutative diagram

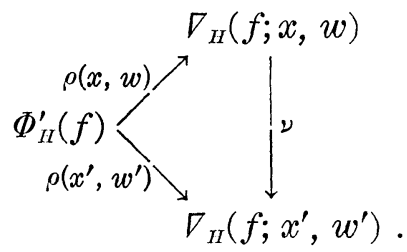

The conclusions (c) and (d) show that $\nu$ is bijective and that we can identify all $\nabla_{I I}(f ; x, w)$ by $\nu$ to get an abstract set denoted by $\nabla_{I I}(f)$. Then (e) shows that we can identify all $\rho(x, w)$ to get an injection $\rho: \Phi_{I I}^{\prime}(f) \rightarrow \nabla_{I I}(f)$.

The elements of $\nabla_{I I}(f)$ are called fixed point $H$-classes of $f . \mathrm{A}$ 
pair $(x, w)$ with $x \in X$ and $w$ a path from $x$ to $f(x)$ is called a reference pair for $f$. Each element of $\nabla_{H}(f ; x, w)$ is called the representation of the corresponding element of $\nabla_{H}(f)$ in the reference pair $(x, w)$. We will identify each element $\boldsymbol{F}$ of $\Phi_{I I}^{\prime}(f)$ with $\rho(\boldsymbol{F})$, and think of $\Phi_{H}^{\prime}(f)$ as a subset of $\nabla_{H}(f)$.

When $H$ is trivial, then the fixed point $H$-classes are the ordinary fixed point classes, and $f_{H}=f_{\pi}, w_{H}=w_{*}, \widetilde{f}_{H}=\widetilde{f}_{\pi}$. In this case, we abbreviate $\nabla_{H}(f)$ to $\nabla(f), \Phi_{H}^{\prime}(f)$ to $\Phi^{\prime}(f), \stackrel{\boldsymbol{H}}{\simeq}$ to $\simeq$ and so on.

We now state some results about the fixed point $H$-classes without proof.

Let $G: f \simeq g: X \rightarrow X$ be a homotopy, where $f$ satisfies $f_{\pi}(H) \subset H$ (so $g_{\pi}(H) \subset H$ ). The following lemma shows the relation between $\nabla_{H}(f)$ and $\nabla_{H}(g)$.

LEMMA 1.3. (a) Let $(x, w)$ be a reference pair for $f$, and $\left(x^{\prime}, w^{\prime}\right)$ be a reference pair for $g$. Let $u$ be a path from $x$ to $x^{\prime}$. If $\langle a\rangle_{H}$, $\left\langle a^{\prime}\right\rangle_{H} \in \nabla_{H}(f ; x, w)$ are $\sim$ equivalent, then

$$
\left\langle u^{-1} a w \Delta(G, u) w^{\prime-1}\right\rangle_{H} \sim\left\langle u^{-1} a^{\prime} w \Delta(G, u) w^{\prime-1}\right\rangle_{H},
$$

where $\Delta(G, u)$ is the diagonal path defined by $\Delta(G, u)(t)=G(u(t), t)$. Thus we can define a transformation $\mu_{G}: \nabla_{H}(f ; x, w) \rightarrow \nabla_{H}\left(g ; x^{\prime}, w^{\prime}\right)$ by

$$
\mu_{G}\left(\left[\langle a\rangle_{H}\right]\right)=\left[\left\langle u^{-1} a w \Delta(G, u) w^{\prime-1}\right\rangle_{I I}\right] .
$$

Since $\nabla_{H}(f ; x, w)$ and $\nabla_{H}\left(g ; x^{\prime}, w^{\prime}\right)$ are representations of $\nabla_{H}(f)$ and $\nabla_{H}(g)$ respectively, we can think of $\mu_{G}$ as a transformation from $\nabla_{H}(f)$ to $\nabla_{H}(f)$.

(b) $\mu_{G}$ does not depend on the pairs $(x, w),\left(x^{\prime}, w^{\prime}\right)$ and the path $u$.

(c) $\mu_{G}$ is a bijection.

(d) Let $x_{0} \in \boldsymbol{F}_{0} \in \Phi_{H}^{\prime}(f)$ and $x_{1} \in \boldsymbol{F}_{1} \in \Phi_{H}^{\prime}(g)$. Then $\mu_{G}\left(\boldsymbol{F}_{0}\right)=\boldsymbol{F}_{1}$ if and only if there is a path $c$ from $x_{0}$ to $x_{1}$ such that $c \stackrel{\boldsymbol{H}}{\simeq} \Delta(G, c)$.

REMARK. Since the representation (2) of $\mu_{G}$ depends on the pairs $(x, w),\left(x^{\prime}, w^{\prime}\right)$ and the path $u$, we may get a very simple representation by choosing suitable pairs and path. We will frequently use $x^{\prime}=x, w^{\prime}=w G_{x}$ (where $G_{x}$ is the trace of $G$ at $x$, that is, the path defined by $\left.G_{x}(t)=G(x, t)\right)$, and $u$ is the constant path at $x$. Then $\Delta(G, u)=G_{x}$ and (2) becomes

$$
\mu_{G}\left(\left[\langle a\rangle_{H}\right]\right)=\left[\langle a\rangle_{H}\right] \text {. }
$$

(Note that the $\left[\langle a\rangle_{H}\right]$ on the left hand side is in $\nabla_{H}(f ; x, w)$ and the other one is in $\nabla_{H}\left(g ; x^{\prime}, w^{\prime}\right)$.)

Let $X, Y$ be spaces and let $f: X \rightarrow X, g: Y \rightarrow Y$ and $h: X \rightarrow Y$ be maps so that the diagram 


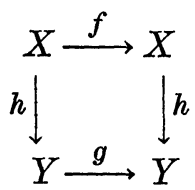

is commutative. Then we have $h(\Phi(f)) \subset \Phi(g)$. Furthermore, if $H$ and $H^{\prime}$ are normal subgroups of $\pi_{1}(X)$ and $\pi_{1}(Y)$ respectively, and $f_{\pi}(H) \subset H, g_{\pi}\left(H^{\prime}\right) \subset H^{\prime}, h_{\pi}(H) \subset H^{\prime}$, then we have

Lemma 1.4. (a) If $(x, w)$ is a reference pair for $f$, then $(h(x)$, $h \circ w)$ is a reference pair for $g$. We can define a transformation $h_{\nabla}: \nabla_{H}(f ; x, w) \rightarrow \nabla_{H^{\prime}}(g ; h(x), h \circ w)$ by

$$
h_{\nabla}\left(\left[\langle a\rangle_{H}\right]\right)=\left[\langle h \circ a\rangle_{H^{\prime}}\right] .
$$

Thus we get $h_{\nabla}: \nabla_{H}(f) \rightarrow \nabla_{H^{\prime}}(g)$.

(b) $h_{\nabla}$ is independent of the choice of $(x, w)$.

(c) If $x^{\prime} \in \boldsymbol{F} \in \Phi_{H}^{\prime}(f)$, then $h\left(x^{\prime}\right) \in h_{\nabla}(\boldsymbol{F}) \in \Phi_{H^{\prime}}^{\prime}(g)$.

(d) If $Z$ is another space, $H^{\prime \prime}$ is a normal subgroup of $\pi_{1}(Z)$, and $k: Y \rightarrow Z, \iota: Z \rightarrow Z$ are maps such that $k \circ g=\ell \circ k, k\left(H^{\prime}\right) \subset H^{\prime \prime}$, $\ell\left(H^{\prime \prime}\right) \subset H^{\prime \prime}$, then we have $(k \circ h)_{\nabla}=k_{\nabla} \circ h_{\nabla}$.

Corollary 1.5. Let $X, Y, H$ and $H^{\prime}$ be as above. Suppose that $h: X \rightarrow Y$ and $h^{\prime}: Y \rightarrow X$ are maps such that $h_{\pi}(H) \subset H^{\prime}, h_{\pi}^{\prime}\left(H^{\prime}\right) \subset H$. Let $f=h^{\prime} \circ h: X \rightarrow X$ and $g=h \circ h^{\prime}: Y \rightarrow Y$. ( $f, g$ are called a pair of commuting maps.) Then the diagrams
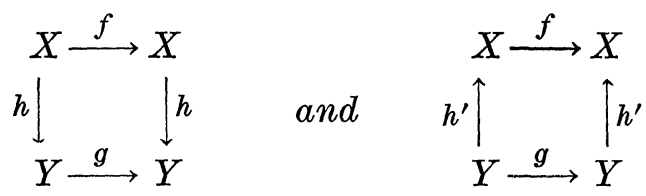

are commutative, and $h_{\nabla}:\left(\nabla_{H}(f), \Phi_{H}^{\prime}(f)\right) \rightarrow\left(\nabla_{H^{\prime}}(g), \Phi_{H}^{\prime}(g)\right), h_{V}^{\prime}:\left(\nabla_{H^{\prime}}(g)\right.$, $\left.\Phi_{H}^{\prime}(g)\right) \rightarrow\left(\nabla_{H}(f), \Phi_{H}^{\prime}(f)\right)$ are defined. Moreover $h_{\nabla}^{\prime} \circ h_{\nabla}$ and $h_{\nabla} \circ h_{\nabla}^{\prime}$ are both the identities, so $h_{\nabla}$ is a bijection with inverse $h_{\nabla}^{\prime}$.

CoRollaRY 1.6. Let $H, H^{\prime}$ be two normal subgroups of $\pi_{1}(X)$ such that $H^{\prime} \subset H$. Then for any map $f: X \rightarrow X$ satisfying $f_{\pi}(H) \subset H$ and $f_{\pi}\left(H^{\prime}\right) \subset H^{\prime}$, we have the transformation $\operatorname{id}_{\nabla}:\left(\nabla_{H^{\prime}}(f), \Phi_{H^{\prime}}^{\prime}(f)\right) \rightarrow$ $\left(\nabla_{H}(f), \Phi_{H}^{\prime}(f)\right)$, where id is the identity map of $X$. In particular, we have $\mathrm{id}_{\nabla}:\left(\nabla(f), \Phi^{\prime}(f)\right) \rightarrow\left(\nabla_{H}(f), \Phi_{H}^{\prime}(f)\right)$.

Note that $\mathrm{id}_{\nabla}$ is surjective.

2. Fixed point classes of a fiber map. The transformation $T_{\bar{w}}$. From this section on, let $\lambda$ be a regular lifting function of a 
fiber space $(E, p, B)$ with $E, B$ and all fibers path-connected. For any path $\bar{c}$ in $B$ from $b$ to $b^{\prime}$, we define a map (translation) $\tau_{\bar{c}}: p^{-1}(b) \rightarrow$ $p^{-1}\left(b^{\prime}\right)$ by $\tau_{\bar{c}}(x)=\lambda(x, \bar{c})(1)$. Note that if $\bar{c}$ is a constant path, then $\tau_{\bar{c}}=\mathrm{id}$.

Lemma 2.1. For any path $d$ in $p^{-1}(b)$, we have in $E$

$$
\tau_{\bar{c}} \circ d \simeq \lambda(d(0), \bar{c})^{-1} d \lambda(d(1), \bar{c}) .
$$

Proof. We define a map $H: I \times I \rightarrow E$ by

$$
H(t, s)=\lambda(d(t), \bar{c})(s) .
$$

The restrictions of $H$ to the four sides of the square $I \times I$ are paths $\lambda(d(0), \bar{c}), \lambda(d(1), \bar{c}), d$ and $\tau_{\bar{c}} \circ d$. From this we get the conclusion of the lemma.

For each $b \in B$ and $x \in p^{-1}(b)$, let $K_{b}(x)$ denote the kernel of the homomorphism $\left(i_{b}\right)_{\pi}: \pi_{1}\left(p^{-1}(b), x\right) \rightarrow \pi_{1}(E, x)$, where $i_{b}: p^{-1}(b) \rightarrow E$ is the inclusion. Then $K_{b}(x)$ is a normal subgroup of $\pi_{1}\left(p^{-1}(b), x\right)$. For any path $\zeta$ in $p^{-1}(b)$ from $x$ to $x^{\prime}$, it is easy to prove $\ell_{*}\left(K_{b}(x)\right)=$ $K_{b}\left(x^{\prime}\right)$. Thus we get a normal subgroup of $\pi_{1}\left(p^{-1}(b)\right)$ denoted by $K_{b}$ (or $K$ briefly). Note that for two paths $d$ and $d^{\prime}$ in $p^{-1}(b), d \stackrel{\boldsymbol{K}}{\simeq} d^{\prime}$ if and only if $d \simeq d^{\prime}$ in $E$.

Let $\bar{c}$ be a path in $B$ from $b$ to $b^{\prime}$, and let $x \in p^{-1}(b), x^{\prime}=\tau_{\bar{c}}(x)$. It follows from Lemma 2.1 that the diagram

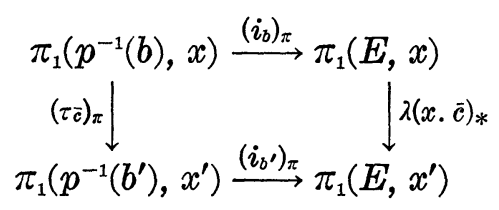

is commutative. Since $\left(\tau_{\bar{c}}\right)_{\pi}$ and $\lambda(x, \bar{c})_{*}$ are both isomorphisms, we get $\left(\tau_{\bar{c}}\right)_{\pi}\left(K_{b}(x)\right)=K_{b^{\prime}}\left(x^{\prime}\right)$. Thus $\left(\tau_{\bar{c}}\right)_{\pi}\left(K_{b}\right)=K_{b^{\prime}}$.

Let $f: E \rightarrow E$ be a fiber map inducing $\bar{f}: B \rightarrow B$, let $b \in \Phi(f)$ and let $f_{b}$ be the restriction of $f$ to $p^{-1}(b)$. For any $x \in p^{-1}(b)$, the commutative diagram

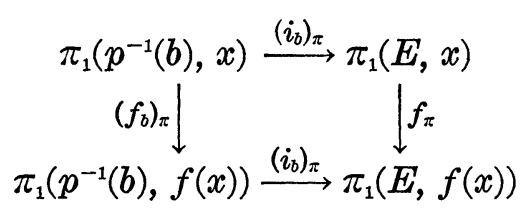

implies $\left(f_{b}\right)_{\pi}\left(K_{b}(x)\right) \subset K_{b}(f(x))$, so $\left(f_{b}\right)_{\pi}\left(K_{b}\right) \subset K_{b}$ and $\nabla_{K}\left(f_{b}\right), \Phi_{K}^{\prime}\left(f_{b}\right)$ are defined.

Let $F=\left\{f_{t}\right\}: E \times I \rightarrow E$ be a fiber homotopy from $f$ to $g$, and 
let $\bar{F}=\left\{\bar{f}_{t}\right\}: B \times I \rightarrow B$ be the homotopy induced by $F$. Let $b \in \Phi(\bar{f})$, $b^{\prime} \in \Phi(\bar{g})$ and let $\bar{w}$ be a path in $B$ from $b$ to $b^{\prime}$ such that $\Delta(\bar{F}, \bar{w}) \simeq \bar{w}$.

LEMmA 2.2. There is a bijection $T_{\bar{w}}$ from $\nabla_{K}\left(f_{b}\right)$ to $\nabla_{K}\left(g_{b^{\prime}}\right)$.

Proof. Define maps

$$
\begin{aligned}
& h=\tau_{\Delta(\bar{F}, \bar{w})^{-1}} \circ g_{b^{\prime}} \circ \tau_{\bar{w}}: p^{-1}(b) \longrightarrow p^{-1}(b), \\
& h^{\prime}=\tau_{\bar{w}} \circ \tau_{\Delta(\bar{F}, \bar{w})^{-1}} \circ g_{b^{\prime}}: p^{-1}\left(b^{\prime}\right) \longrightarrow p^{-1}\left(b^{\prime}\right), \\
& h^{\prime \prime}=\tau_{\Delta(\bar{F}, \bar{w})} \circ \tau_{\Delta(\bar{F}, \bar{w})^{-1}} \circ g_{b^{\prime}}: p^{-1}\left(b^{\prime}\right) \longrightarrow p^{-1}\left(b^{\prime}\right) .
\end{aligned}
$$

The maps $h$ and $h^{\prime}$ are a pair of commuting maps. Since $\left(\tau_{\bar{w}}\right)_{\pi}\left(K_{b}\right)=$ $K_{b^{\prime}}$ and $\left(\tau_{\Delta(\bar{F}, \bar{w})^{-1}} \circ g_{b^{\prime}}\right)_{\pi}\left(K_{b^{\prime}}\right) \subset\left(\tau_{\left.\Delta(\bar{F}, \bar{w})^{-1}\right)_{\pi}}\left(K_{b^{\prime}}\right)=K_{b}\right.$, by Corollary 1.5, we have a bijection $\left(\tau_{\bar{w}}\right)_{\nabla}: \nabla_{K}(h) \rightarrow \nabla_{K}\left(h^{\prime}\right)$.

For a path $c$, let $c_{r}^{s}(r, s \in I)$ denote the subpath of $c$ defined by $c_{r}^{s}(t)=c(r+(s-r) t)$.

Let $D=\left\{d_{t}\right\}: \bar{w} \simeq \Delta(\bar{F}, \bar{w})$. We construct homotopies $H: p^{-1}(b) \times$ $I \rightarrow p^{-1}(b)$ and $H^{\prime}, H^{\prime \prime}: p^{-1}\left(b^{\prime}\right) \times I \rightarrow p^{-1}\left(b^{\prime}\right)$ by

$$
\begin{aligned}
& H(x, t)=\tau_{\Delta(\bar{F}, \bar{w})_{t}^{0}}(\Delta(F, \lambda(x, \bar{w}))(t)), \\
& H^{\prime}\left(x^{\prime}, t\right)=\tau_{d_{t}} \circ \tau_{\Delta(\bar{F}, \bar{w})^{-1}} \circ g_{b^{\prime}}\left(x^{\prime}\right), \\
& H^{\prime \prime}\left(x^{\prime}, t\right)=\tau_{\Delta(\bar{F}, \bar{w})_{t}^{1}}\left(\lambda\left(g_{b^{\prime}}\left(x^{\prime}\right), \Delta(\bar{F}, \bar{w})^{-1}\right)(1-t)\right) .
\end{aligned}
$$

Then $H$ is from $f_{b}$ to $h, H^{\prime}$ is from $h^{\prime}$ to $h^{\prime \prime}$ and $H^{\prime \prime}$ is from $h^{\prime \prime}$ to $g_{b^{\prime}}$. By Lemma 1.3, we have bijections $\mu_{H}, \mu_{H^{\prime}}$ and $\mu_{H^{\prime \prime}}$. Let

$$
T_{\bar{w}}=\mu_{H^{\prime \prime}} \circ \mu_{H^{\prime}} \circ\left(\tau_{\bar{w}}\right)_{\nabla} \circ \mu_{H}: \nabla_{K}\left(f_{b}\right) \longrightarrow \nabla_{K}\left(g_{b^{\prime}}\right),
$$

then $T_{\bar{w}}$ is a bijection.

In calculation we always use a representation of $T_{\bar{w}}$. We can indeed get an extremely simple representation by a special choice of reference pairs. Thus let $(x, r)$ be a pair for $f_{b}$. The special choice for $h$ is $\left(x, r H_{x}\right.$ ) (cf. the remark following Lemma 1.3); the special choice for $h^{\prime}$ is $\left(x^{\prime}, \tau_{\bar{w}} \circ\left(r H_{x}\right)\right.$ ), where $x^{\prime}=\tau_{\bar{w}}(x)$ (cf. Lemma 1.4); the special choice for $h^{\prime \prime}$ is $\left(x^{\prime},\left(\tau_{\bar{w}} \circ\left(r H_{x}\right)\right) H_{x^{\prime}}^{\prime}\right)$, and the special choice for $g_{b^{\prime}}$ is $\left(x^{\prime},\left(\tau_{\bar{w}} \circ\left(r H_{x}\right)\right) H_{x^{\prime}}^{\prime} H_{x^{\prime}}^{\prime \prime}\right)$, where $H_{x}, H_{x^{\prime}}^{\prime}, H_{x^{\prime}}^{\prime \prime}$ are traces. Let $\boldsymbol{r}^{\prime}=\left(\tau_{\bar{w}} \circ\left(r H_{x}\right)\right) H_{x^{\prime}}^{\prime} H_{x^{\prime}}^{\prime \prime}$. Then $\left(x^{\prime}, \boldsymbol{r}^{\prime}\right)$ is called the induced pair from $(x, r)$ and $\bar{w}$. By (2a) and (3), $T_{\bar{w}}: \nabla_{K}\left(f_{b} ; x, r\right) \rightarrow \nabla_{K}\left(g_{b^{\prime}} ; x^{\prime}, r^{\prime}\right)$ is given by

$$
T_{\bar{w}}\left(\left[\langle a\rangle_{K}\right]\right)=\left[\left\langle\tau_{\bar{w}} \circ a\right\rangle_{K}\right]
$$

LEMMA 2.3. Let $(x, r)$ be a reference pair for $f_{b}$, and let $\left(x^{\prime}, r^{\prime}\right)$ be the induced pair for $g_{b}^{\prime}$ from $(x, r)$ and $\bar{w}$. Let $w=\lambda(x, \bar{w})$, then in $E$ 


$$
r^{\prime} \simeq w^{-1} r \Delta(F, w)
$$

Proof. We construct maps $G_{i}: I \times I \rightarrow E, i=1,2,3$, by

$$
\begin{aligned}
& G_{1}(t, s)=\lambda\left(\Delta(F, w)(t), \Delta(\bar{F}, \bar{w})_{t}^{0}\right)(s), \\
& G_{2}(t, s)=\lambda\left(h(x), d_{t}\right)(s), \\
& G_{3}(t, s)=\lambda\left(\lambda\left(g_{b^{\prime}}\left(x^{\prime}\right), \Delta(\bar{F}, \bar{w})^{-1}\right)(1-t), \Delta(\bar{F}, \bar{w})_{t}^{1}\right)(s) .
\end{aligned}
$$

Calculating the paths defined by restricting the $G_{i}$ to the four sides of $I \times I$, we get in $E$

$$
\begin{aligned}
& H_{x} \simeq \Delta(F, w) \lambda\left(g_{b^{\prime}}\left(x^{\prime}\right), \Delta(\bar{F}, \bar{w})^{-1}\right), \\
& H_{x^{\prime}}^{\prime \prime} \simeq \lambda(h(x), \bar{w})^{-1} \lambda(h(x), \Delta(\bar{F}, \bar{w})) \\
& H_{x^{\prime}}^{\prime \prime} \simeq \lambda(h(x), \Delta(\bar{F}, \bar{w}))^{-1} \lambda\left(g_{b^{\prime}}\left(x^{\prime}\right), \Delta(\bar{F}, \bar{w})^{-1}\right) .
\end{aligned}
$$

By Lemma 2.1, we have in $E$

$$
\tau_{\bar{w}} \circ\left(r H_{x}\right) \simeq w^{-1} r H_{x} \lambda(h(x), \bar{w}) .
$$

Applying the four formulas to the definition of $r^{\prime}$, we get (5).

Note that $w^{-1} r \Delta(F, w)$ does not depend on $D$, so $\left\langle r^{\prime}\right\rangle_{K}$ does not depend on $D$. Moreover, the representation of $T_{\bar{w}}$ in $(x, r)$ and $\left(x^{\prime}, r^{\prime}\right)$ does not depend on $D$ either. By (c) of Lemma 1.2, we get:

CoROLlary 2.4. The function $T_{\bar{w}}$ does not depend on the choice of the homotopy $D$ used in its construction.

Lemma 2.5. The diagram

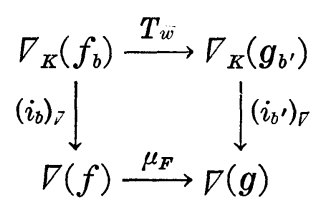

is commutative.

Proof. We need only to prove the diagram

$$
\begin{gathered}
\nabla_{K}\left(f_{b} ; x, r\right) \stackrel{T_{w}}{\longrightarrow} \nabla_{K}\left(g_{b^{\prime}} ; x^{\prime}, r^{\prime}\right) \\
\quad\left(i_{b}\right)_{\nu} \downarrow \\
\left.\nabla(f ; x, r) \stackrel{\mu_{F}}{\longrightarrow} \nabla\left(g ; i^{\prime}\right)_{V}, r^{\prime}\right)
\end{gathered}
$$

is commutative, where $(x, r)$ is a reference pair for $f_{b}$ and $\left(x^{\prime}, r^{\prime}\right)$ is induced from $(x, r)$ and $\bar{w}$. For any $\left[\langle a\rangle_{K}\right] \in \nabla_{K}\left(f_{b} ; x, r\right)$, 


$$
\begin{aligned}
\left(i_{b^{\prime}}\right)_{\nabla} \circ T_{\bar{w}}\left(\left[\langle a\rangle_{K}\right]\right) & =\left(i_{b^{\prime}}\right)_{V}\left(\left[\left\langle\tau_{\bar{w}} \circ a\right\rangle_{K}\right]\right) & & (\text { by }(4)) \\
& =\left[\left\langle\tau_{\bar{w}} \circ a\right\rangle\right] & & (\text { by (3)) } \\
& =\left[\left\langle w^{-1} a w\right\rangle\right] & & (\text { Lemma 2.1). }
\end{aligned}
$$

On the other hand, since $w$ is a path from $x$ to $x^{\prime}$,

$$
\begin{aligned}
\mu_{F} \circ\left(i_{b}\right)_{V}\left(\left[\langle a\rangle_{K}\right]\right) & =\mu_{F}([\langle a\rangle]) & & (\text { by (3)) } \\
& =\left[\left\langle w^{-1} a r \Delta(F, w) r^{\prime-1}\right\rangle\right] & & (\text { by (2)) } \\
& =\left[\left\langle w^{-1} a w\right\rangle\right] & & \text { (by (5)). }
\end{aligned}
$$

We will often apply 2.2-2.5 in a special case, namely when $\boldsymbol{F}$ is a constant homotopy $f_{t} \equiv f$. In this case, $b, b^{\prime}$ are in the same class of $\bar{f}, \Delta(\bar{F}, \bar{w})=\bar{f} \circ \bar{w}, \Delta(F, w)=f \circ w,(5)$ becomes

$$
r^{\prime} \simeq w^{-1} r(f \circ w)
$$

and the diagram in Lemma 2.5 becomes

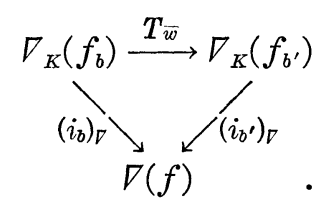

We now add two properties of $T_{\bar{w}}$ in this special case. Before this, we first prove

LEMMA 2.6. Let $v$ be a path in $E$ such that $v(0), v(1) \in p^{-1}(b)$ and $\langle p \circ v\rangle$ is the unit of $\pi_{1}(B, b)$. Then there is a path $\ell$ in $p^{-1}(b)$ from $v(0)$ to $v(1)$ such that $\ell \simeq v$ in $E$.

Proof. We first take an arbitrary path $\ell^{\prime}$ in $p^{-1}(b)$ from $v(0)$ to $v(1)$. Then $v \ell^{\prime-1}$ is a loop based at $v(0)$, and $p_{\pi}\left(\left\langle v \ell^{\prime-1}\right\rangle\right)$ is the unit. By the exactness of the sequence

$$
\pi_{1}\left(p^{-1}(b), v(0)\right) \stackrel{\left(i_{b}\right)_{\pi}}{\longrightarrow} \pi_{1}(E, v(0)) \stackrel{p_{\pi}}{\longrightarrow} \pi_{1}(B, b)
$$

we can find an element $\gamma$ of $\pi_{1}\left(p^{-1}(b), v(0)\right)$ such that $\left(i_{b}\right)_{\pi}(\gamma)=\left\langle v \ell^{\prime-1}\right\rangle$. Let $u \in \gamma$. Then, in $E, u \simeq v \ell^{\prime-1}$, and the path $\ell=u \ell^{\prime}$ meets the need of the lemma.

Lemma 2.7. If $\bar{w}^{\prime} \simeq \bar{w}$, then $T_{\bar{w}^{\prime}}=T_{w}$.

Proof. Let $(x, r)$ be a reference pair for $f_{b}$, let $\left(x^{\prime}, r^{\prime}\right)$ be the induced pair for $f_{b^{\prime}}$ from $(x, r)$ and $\bar{w}$, and let $\left(x^{\prime \prime}, r^{\prime \prime}\right)$ be the induced pair for $f_{b^{\prime}}$ from $(x, r)$ and $\bar{w}^{\prime}$. Let $w=\lambda(x, \bar{w}), w^{\prime}=\lambda\left(x, \bar{w}^{\prime}\right)$. We need only to prove that the diagram 


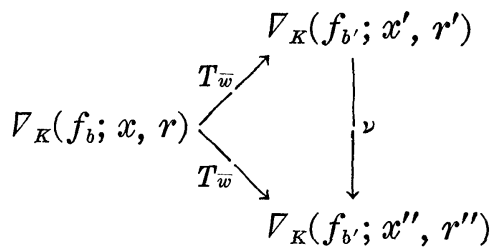

is commutative. Since $\left\langle p \circ\left(w^{-1} w^{\prime}\right)\right\rangle$ is the unit of $\pi_{1}\left(B, b^{\prime}\right)$, we can find a path $\ell$ in $p^{-1}\left(b^{\prime}\right)$ from $x^{\prime}$ to $x^{\prime \prime}$ such that $\ell \simeq w^{-1} w^{\prime}$ (Lemma 2.6). For any $\left[\langle a\rangle_{K}\right] \in \nabla_{K}\left(f_{b} ; x, r\right)$, we have

$$
\begin{aligned}
\nu \circ T_{\bar{w}}\left(\left[\langle a\rangle_{K}\right]\right) & =\nu\left(\left[\left\langle\tau_{\bar{w}} \circ a\right\rangle_{K}\right]\right) \\
& =\left[\left\langle\ell^{-1}\left(\tau_{\bar{w}} \circ a\right) r^{\prime}(f \circ \ell) r^{\prime \prime-1}\right\rangle_{K}\right]
\end{aligned}
$$

Since in $E$ (by (5a) and Lemma 2.1)

$$
\begin{aligned}
\ell^{-1}\left(\tau_{\bar{i}} \circ a\right) r^{\prime}(f \circ \ell) r^{\prime \prime-1} & \simeq w^{\prime-1} w w^{-1} a w w^{-1} r(f \circ w)\left(f \circ w^{-1} w^{\prime}\right)\left(f \circ w^{\prime-1}\right) r^{-1} w^{\prime} \\
& \simeq w^{\prime-1} a w^{\prime} \simeq \tau_{\bar{w}} \circ a
\end{aligned}
$$

so

$$
\left\langle\ell^{-1}\left(\tau_{\bar{w}} \circ a\right) r^{\prime}(f \circ \ell) r^{\prime \prime-1}\right\rangle_{K}=\left\langle\tau_{\bar{w}}, \circ\right\rangle_{K}
$$

and

$$
\nu \circ T_{\bar{w}}\left(\left[\langle a\rangle_{K}\right]\right)=\left[\left\langle\tau_{\bar{w}^{\prime}} \circ a\right\rangle_{K}\right]=T_{\bar{w}^{\prime}}\left(\left[\langle a\rangle_{K}\right]\right) .
$$

Similarly, we can prove

LEMma 2.8. Let $b, b^{\prime}, b^{\prime \prime} \in \Phi(\bar{f})$ be in the same class, and $\bar{w}^{\prime}$ be $a$ path from $b$ to $b^{\prime}, \bar{w}^{\prime \prime}$ from $b^{\prime}$ to $b^{\prime \prime}$ such that $\bar{w}^{\prime} \simeq \bar{f} \circ \bar{w}^{\prime}$ and $\bar{w}^{\prime \prime} \simeq \bar{f} \circ \bar{w}^{\prime \prime}$. Let $\bar{w}=\bar{w}^{\prime} \bar{w}^{\prime \prime}$. Then

$$
T_{\bar{w}}=T_{\bar{w}^{\prime}, \circ} \circ T_{\bar{w}^{\prime}} .
$$

3. The structure of fixed point classes of a fiber map. Let $(E, p, B)$ be a fiber space with $E, B$ and all fibers path-connected, and let $f: E \rightarrow E$ be a fiber map. Since $p \circ f=\bar{f} \circ p$ and $i_{b} \circ f_{b}=f \circ i_{b}$, by Lemma 1.4 , we can define $p_{\nabla}:\left(\nabla(f), \Phi^{\prime}(f)\right) \rightarrow\left(\nabla(\bar{f}), \Phi^{\prime}(\bar{f})\right)$ and $\left(i_{b}\right)_{i}:\left(\nabla_{K}\left(f_{b}\right), \Phi_{K}^{\prime}\left(f_{b}\right)\right) \rightarrow\left(\nabla(f), \Phi^{\prime}(f)\right)$ (for any $\left.b \in \Phi(\bar{f})\right)$. Note that for any $\boldsymbol{F} \in \Phi_{K}^{\prime}\left(f_{b}\right)$, the fixed point class $p_{\nabla} \circ\left(i_{b}\right)_{\nabla}(\boldsymbol{F}) \in \Phi^{\prime}(\bar{f})$ contains $b$.

THEOREM 3.1. Let $b, b^{\prime} \in \Phi(\bar{f})$, and $\boldsymbol{F}_{0} \in \nabla_{K}\left(f_{b}\right), \boldsymbol{F}_{0}^{\prime} \in \nabla_{K}\left(f_{b^{\prime}}\right)$. Then $\left(i_{b}\right)_{\bar{v}}\left(\boldsymbol{F}_{0}\right)=\left(i_{b^{\prime}}\right)_{V}\left(\boldsymbol{F}_{0}^{\prime}\right)$ if and only if there is a path $\bar{w}$ in $B$ from $b$ to $b^{\prime}$ such that $\bar{w} \simeq \bar{f} \circ \bar{w}$ and $T_{\bar{w}}\left(\boldsymbol{F}_{0}\right)=\boldsymbol{F}_{0}^{\prime}$.

Proof. "If" is implied by Lemma 2.5. We now prove "only if".

Let $(x, r)$ and $\left(x^{\prime}, r^{\prime}\right)$ be reference pairs for $f_{b}$ and $f_{b^{\prime}}$, respectively. Suppose $\left[\langle a\rangle_{K}\right]$ is the representation of $\boldsymbol{F}_{0}$ in $(x, r)$ and 
$\left[\left\langle a^{\prime}\right\rangle_{K}\right]$ is the representation of $\boldsymbol{F}_{0}^{\prime}$ in $\left(x^{\prime}, r^{\prime}\right)$. Since $\left(i_{b}\right)_{\nabla}\left(\boldsymbol{F}_{0}\right)=\left(i_{b^{\prime}}\right),\left(\boldsymbol{F}_{0}^{\prime}\right) \epsilon$ $\nabla(f)$, its representation is $[\langle a\rangle]$ in $(x, r)$ and is $\left[\left\langle a^{\prime}\right\rangle\right]$ in $\left(x^{\prime}, r^{\prime}\right)$. Let $v^{\prime}$ be a path in $E$ from $x$ to $x^{\prime}$, then by $(1),\left[\left\langle a^{\prime}\right\rangle\right]=\left[\left\langle v^{\prime-1} a r\left(f \circ v^{\prime}\right) r^{\prime-1}\right\rangle\right]$. Thus we can find $\langle u\rangle \in \pi_{1}\left(E, x^{\prime}\right)$ such that in $E$

$$
\begin{aligned}
\left\langle a^{\prime}\right\rangle & =\langle u\rangle\left\langle v^{\prime-1} a r\left(f \circ v^{\prime}\right) r^{\prime-1}\right\rangle\left(r_{*}^{\prime-1} \circ f_{\pi}\left(\langle u\rangle^{-1}\right)\right) \\
& =\left\langle u v^{\prime-1} \operatorname{ar}\left(f \circ\left(v^{\prime} u^{-1}\right)\right) r^{\prime-1}\right\rangle .
\end{aligned}
$$

Let $v=v^{\prime} u^{-1}$ and $\bar{w}=p \circ v$. Then the above formula implies $\bar{w} \simeq$ $\bar{f} \circ \bar{w}$.

It remains to prove $T_{w}\left(\boldsymbol{F}_{0}\right)=\boldsymbol{F}_{0}^{\prime}$. To do it, we calculate the representation of $T_{\ddot{w}}\left(\boldsymbol{F}_{0}\right)$ in $\left(x^{\prime}, r^{\prime}\right)$. Suppose $\left(x^{\prime \prime}, r^{\prime \prime}\right)$ is the pair for $f_{b^{\prime}}$ induced from $(x, r)$ and $\bar{w}$. By $(4)$, the representation of $T_{\bar{w}}\left(\boldsymbol{F}_{0}\right)$ in $\left(x^{\prime \prime}, r^{\prime \prime}\right)$ is $\left[\left\langle\tau_{\bar{w}} \circ a\right\rangle_{K}\right]$. Let $w=\lambda(x, \bar{w})$. Since $\left\langle p \circ\left(w^{-1} v\right)\right\rangle$ is the unit of $\pi_{1}\left(B, b^{\prime}\right)$, we can find a path $\ell$ in $p^{-1}\left(b^{\prime}\right)$ from $x^{\prime \prime}$ to $x^{\prime}$ such that $\ell \simeq w^{-1} v$ in $E$ (Lemma 2.6). Then by (1), the representation of $T_{w}\left(\boldsymbol{F}_{0}\right)$ in $\left(x^{\prime}, r^{\prime}\right)$ is $\left[\left\langle\ell^{-1}\left(\tau_{w} \circ a\right) r^{\prime \prime}(f \circ \ell) r^{\prime-1}\right\rangle_{K}\right]$. By Lemma 2.1 and (5a), in $E$

$$
\begin{aligned}
\ell^{-1}\left(\tau_{\bar{w}} \circ a\right) r^{\prime \prime}(f \circ \ell) r^{\prime-1} & \simeq v^{-1} w w^{-1} a w w^{-1} r(f \circ w)(f \circ \ell) r^{\prime-1} \\
& \simeq v^{-1} a r(f \circ v) r^{\prime-1} \simeq a^{\prime}
\end{aligned}
$$

so $\left\langle\ell^{-1}\left(\tau_{\bar{w}} \circ \alpha\right) \boldsymbol{r}^{\prime \prime}(f \circ \ell) \boldsymbol{r}^{\prime-1}\right\rangle_{K}=\left\langle a^{\prime}\right\rangle_{K}$ and $T_{w}\left(\boldsymbol{F}_{0}\right)=\left[\left\langle a^{\prime}\right\rangle_{K}\right]=\boldsymbol{F}_{0}^{\prime}$.

Let $X$ be a compact connected ANR, $H$ be a normal subgroup of $\pi_{1}(X)$, and let $f: X \rightarrow X$ be a map such that $f_{\pi}(H) \subset H$. Then for any $\boldsymbol{F} \in \nabla_{H}(f)$ we define its index $i_{f}(\boldsymbol{F})$ as follows: if $\boldsymbol{F} \in \Phi_{H}^{\prime}(f)$, then $i_{f}(\boldsymbol{F})$ is the usual fixed point index (as in [3]), otherwise, $i_{f}(\boldsymbol{F})=0$. A fixed point class $\boldsymbol{F} \in \nabla_{H}(f)$ is said to be essential if $i_{f}(\boldsymbol{F}) \neq 0$. The number of essential fixed point $H$-classes of $f$ is called the $H$-Nielsen number, denoted by $N_{H}(f)$.

Let $G: f \simeq g: X \rightarrow X$, where $f$ has the property $f_{\pi}(H) \subset H$. Then adopting the method used in [3] (cf. p. 98, Theorem 3), we can prove that if $\boldsymbol{F} \in \Phi_{H}^{\prime}(f)$, then

$$
i_{f}(\boldsymbol{F})=\left\{\begin{array}{cl}
i_{g}\left(\mu_{G}(\boldsymbol{F})\right), & \text { if } \mu_{G}(\boldsymbol{F}) \in \Phi_{H}^{\prime}(g), \\
0 & \text { otherwise. }
\end{array}\right.
$$

Thus $\mu_{G}$ preserves index.

Let $X, Y$ be compact connected ANR's, let $H$ and $H^{\prime}$ be normal subgroups of $\pi_{1}(X)$ and $\pi_{1}(Y)$, respectively, and let maps $h: X \rightarrow Y$, $h^{\prime}: Y \rightarrow X$ be such that $h_{\pi}(H) \subset H^{\prime}, h_{\pi}^{\prime}\left(H^{\prime}\right) \subset H$. Let $f=h^{\prime} \circ h, g=$ $h \circ h^{\prime}$. Then by Corollary 1.5, $h_{\nabla}:\left(\nabla_{H}(f), \Phi_{H}^{\prime}(f)\right) \rightarrow\left(\nabla_{H \prime}(g), \Phi_{H}^{\prime}(g)\right)$ is a bijection. By the commutativity of the fixed point index (cf. [3], p. 82), if $\boldsymbol{F} \in \Phi_{H}^{\prime}(f)$, then $i_{f}(\boldsymbol{F})=i_{g}\left(h_{\nabla}(\boldsymbol{F})\right)$. If $\boldsymbol{F} \notin \Phi_{H}^{\prime}(f)$, then $h_{\nabla}(\boldsymbol{F}) \notin$ $\Phi_{I I^{\prime}}^{\prime}(g)$, and $i_{f}(\boldsymbol{F})=0=i_{g}\left(h_{\nu}(\boldsymbol{F})\right)$. Thus we conclude that $h_{\bar{\nu}}$ preserves 
index.

From now on in this paper, let $(E, p, B)$ be a fiber space with $E, B$ and all fibers compact connected ANR's, and let $f: E \rightarrow E$ be a fiber map. From the above discussion, we get

Lemma 3.2. The bijection $T_{i w}$ defined in Lemma 2.2 is indexpreserving.

CoROLlaRY 3.3. If $b, b^{\prime} \in \Phi(\bar{f})$ are in the same class, then $N_{K}\left(f_{b}\right)=N_{K}\left(f_{b^{\prime}}\right)$.

Proof. Let $\bar{w}$ be a path from $b$ to $b^{\prime}$ such that $\bar{w} \simeq \bar{f} \circ \bar{w}$. Then we have $T_{\bar{w}}: \nabla_{K}\left(f_{b}\right) \rightarrow \nabla_{K}\left(f_{b^{\prime}}\right)$, which preserves index (by Lemma 3.2). Thus $N_{K}\left(f_{b}\right)=N_{K}\left(f_{b^{\prime}}\right)$.

From Theorem 3.1 and Lemma 3.2, we get some corollaries.

Let $\# S$ denote the cardinality of a set $S$.

CoRollary 3.4. Let $\boldsymbol{F} \in \nabla(f)$ so that $p_{r}(\boldsymbol{F}) \in \Phi^{\prime}(\bar{f})$. Then for any $b \in p_{\nabla}(\boldsymbol{F})$ the number $\#\left(i_{b}\right)_{\nabla}^{-1}(\boldsymbol{F})$ (i.e., the number of elements in $\left.\left(i_{b}\right)_{\nabla}^{-1}(\boldsymbol{F})\right)$ is a constant, and for any $b \in p_{V}(\boldsymbol{F})$, any $\boldsymbol{F}_{0} \in\left(i_{b}\right)_{V}^{-1}(\boldsymbol{F})$, the index $i_{f_{b}}\left(\boldsymbol{F}_{0}\right)$ is also a constant.

Proof. By Theorem 3.1, for any $b, b^{\prime} \in p_{\nabla}(\boldsymbol{F})$, and for any $\boldsymbol{F}_{0} \in$ $\left(i_{b}\right)_{\nabla}^{-1}(\boldsymbol{F}), \boldsymbol{F}_{0}^{\prime} \in\left(i_{b^{\prime}}\right)_{\nabla}^{-1}(\boldsymbol{F})$, we can find a path $\bar{w}$ in $B$ from $b$ to $b^{\prime}$ such that $\bar{w} \simeq \bar{f} \circ \bar{w}$ and $T_{\bar{w}}\left(\boldsymbol{F}_{0}\right)=\boldsymbol{F}_{0}^{\prime}$. Since $T_{\bar{w}}$ preserves index, then $i_{f_{b}}\left(\boldsymbol{F}_{0}\right)=i_{f_{b^{\prime}}}\left(\boldsymbol{F}_{0}^{\prime}\right)$. Again by Theorem 3.1, $T_{\bar{w}}\left(\left(i_{b}\right)_{\nabla}^{-1}(\boldsymbol{F})\right)=\left(i_{b^{\prime}}\right)_{\bar{v}}^{-1}(\boldsymbol{F})$, so $\#\left(i_{b}\right)_{\nabla}^{-1}(\boldsymbol{F})=\#\left(i_{b^{\prime}}\right)_{\nabla}^{-1}(\boldsymbol{F})$.

DEFINITION 3.5. For each $\boldsymbol{F} \in \nabla(f)$ satisfying $p_{\bar{\nu}}(\boldsymbol{F}) \subset \Phi^{\prime}(\bar{f})$, we define $j(\boldsymbol{F})=i_{f_{b}}\left(\boldsymbol{F}_{0}\right)$ and $k(\boldsymbol{F})=\sharp\left(i_{b}\right)_{\nabla}^{-1}(\boldsymbol{F})$, where $b \in p_{\nabla}(\boldsymbol{F})$ and $\boldsymbol{F}_{0} \in$ $\left(i_{b}\right)_{\nabla}^{-1}(\boldsymbol{F})$.

Corollary 3.6. If $\boldsymbol{F} \in \Phi^{\prime}(f)$ with $j(\boldsymbol{F}) \neq 0$, then

(a) $k(\boldsymbol{F})$ is a positive integer,

(b) as a set, $p_{r}(\boldsymbol{F})=p(\boldsymbol{F})$.

Proof. (a) Since $\boldsymbol{F} \in \Phi^{\prime}(f)$, we know that $p(\boldsymbol{F}) \neq \varnothing$. We take $b \in p(\boldsymbol{F})$. Then $p^{-1}(b) \cap \boldsymbol{F} \neq \varnothing$, so $\left(i_{b}\right)_{\nabla}^{-1}(\boldsymbol{F})$ is nonempty. It follows that $k(\boldsymbol{F})>0$. Since $j(\boldsymbol{F}) \neq 0$, the elements of $\left(i_{b}\right)_{\nabla}^{-1}(\boldsymbol{F})$ are all essential, so $k(\boldsymbol{F})$ is finite.

(b) By Lemma 1.4, one can easily see that $p(\boldsymbol{F}) \subset p_{V}(\boldsymbol{F})$, and we need only prove $p_{\nabla}(\boldsymbol{F}) \subset p(\boldsymbol{F})$. Let $b \in p_{\nu}(\boldsymbol{F})$, then $\boldsymbol{F}$ contains $k(\boldsymbol{F})$ essential fixed point $K$-classes of $f_{b}$, so $\boldsymbol{F} \cap p^{-1}(b) \neq \varnothing$ and $b \in$ 
$p(\boldsymbol{F})$. Thus $p(\boldsymbol{F}) \supset p_{V}(\boldsymbol{F})$.

4. The essential fixed point classes of $f$. In this section we prove

THEOREM 4.1. Suppose $\boldsymbol{F} \in \Phi^{\prime}(f)$. Then $i_{f}(\boldsymbol{F}) \neq 0$ if and only if $j(\boldsymbol{F}) \neq 0$ and $i_{\bar{f}}\left(p_{\nabla}(\boldsymbol{F})\right) \neq 0$.

To prove it, we first give several lemmas.

LEMMA 4.2. Let $F: f \simeq g: E \times I \rightarrow E$ be a fiber homotopy, and let $\quad \boldsymbol{F} \in \Phi^{\prime}(f), \quad \boldsymbol{F}^{\prime}=\mu_{F}(\boldsymbol{F})$. Then $i_{\bar{f}}\left(p_{\bar{V}}(\boldsymbol{F})\right)=i_{\bar{g}}\left(p_{\bar{V}}\left(\boldsymbol{F}^{\prime}\right)\right)$. Suppose further that $p_{\nabla}\left(\boldsymbol{F}^{\prime}\right) \in \Phi^{\prime}(\bar{g})$. Then $j(\boldsymbol{F})=j\left(\boldsymbol{F}^{\prime}\right)$ and $k(\boldsymbol{F})=k\left(\boldsymbol{F}^{\prime}\right)$.

Proof. Let $\bar{F}: \bar{f} \simeq \bar{g}: B \times I \rightarrow B$ be the homotopy induced by $F$. It is easy to check that the diagram

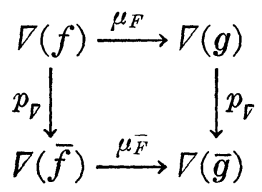

is commutative. Then $\mu_{\bar{F}}\left(p_{\nabla}(\boldsymbol{F})\right)=p_{\nabla}\left(\mu_{F}(\boldsymbol{F})\right)=p_{\nabla}\left(\boldsymbol{F}^{\prime}\right)$, so $i_{\bar{f}}\left(p_{\nabla}(\boldsymbol{F})\right)=$ $i_{\bar{g}}\left(p_{\nu}\left(\boldsymbol{F}^{\prime}\right)\right)$ (because $\mu_{\bar{F}}$ preserves index).

If $p_{\nabla}\left(\boldsymbol{F}^{\prime}\right) \in \Phi^{\prime}(\bar{g})$, we take $b \in p_{\nabla}(\boldsymbol{F})$ and $b^{\prime} \in p_{\nabla}\left(\boldsymbol{F}^{\prime}\right)$. By (d) of Lemma 1.3 , there is a path $\bar{w}$ in $B$ from $b$ to $b^{\prime}$ such that $\bar{w} \simeq \Delta(\bar{F}, \bar{w})$. By Lemma 2.5, $T_{\bar{w}}\left(\left(i_{b}\right)_{\bar{\nabla}}^{-1}(\boldsymbol{F})\right)=\left(i_{b^{\prime}}\right)_{\bar{\nabla}}^{-1}\left(\boldsymbol{F}^{\prime}\right)$. Since $T_{\bar{w}}$ is a bijection and preserves index, we get $j(\boldsymbol{F})=j\left(\boldsymbol{F}^{\prime}\right)$ and $k(\boldsymbol{F})=k\left(\boldsymbol{F}^{\prime}\right)$.

Lemma 4.3. Let $(E, p, B)$ and $\left(E^{\prime}, p^{\prime}, B^{\prime}\right)$ be fiber spaces with $E, E^{\prime}, B, B^{\prime}$ and all fibers compact connected ANR's. Let $h: E \rightarrow E^{\prime}$ and $h^{\prime}: E^{\prime} \rightarrow E$ be fiber maps, and set $f=h^{\prime} \circ h, g=h \circ h^{\prime}$. Suppose $\boldsymbol{F} \in \Phi^{\prime}(f), \boldsymbol{F}^{\prime}=h_{\triangleright}(\boldsymbol{F})$. Then $j(\boldsymbol{F})=j\left(\boldsymbol{F}^{\prime}\right), k(\boldsymbol{F})=k\left(\boldsymbol{F}^{\prime}\right)$ and $i_{\bar{f}}\left(p_{\nabla}(\boldsymbol{F})\right)=$ $i_{\bar{g}}\left(p_{\bar{\nabla}}^{\prime}\left(\boldsymbol{F}^{\prime}\right)\right)$.

Proof. Let maps $\bar{h}: B \rightarrow B^{\prime}$ and $\bar{h}^{\prime}: B^{\prime} \rightarrow B$ be those induced by $h$ and $h^{\prime}$, then $\bar{f}=\bar{h}^{\prime} \circ \bar{h}$ and $\bar{g}=\bar{h} \circ \bar{h}^{\prime}$. Since $h$ is a fiber map, by Lemma 1.4, $p_{\nabla}^{\prime} \circ h_{\nabla}=\left(p^{\prime} \circ h\right)_{\nabla}=(\bar{h} \circ p)_{\nabla}=\bar{h}_{\nabla} \circ p_{\nabla}$. Since $\bar{h}_{\nabla}$ preserves index,

$$
\begin{aligned}
i_{\bar{y}}\left(p_{\nabla}^{\prime}\left(\boldsymbol{F}^{\prime}\right)\right) & =i_{\bar{g}}\left(p_{\nabla}^{\prime} \circ h_{\nabla}(\boldsymbol{F})\right)=i_{\bar{g}}\left(\bar{h}_{\nabla} \circ p_{\nabla}(\boldsymbol{F})\right) \\
& =i_{\bar{f}}\left(p_{\nabla}(\boldsymbol{F})\right)
\end{aligned}
$$

Let $b \in p_{\nabla}(\boldsymbol{F})$. Then $b^{\prime}=\bar{h}(b) \in p_{\nabla}^{\prime}\left(\boldsymbol{F}^{\prime}\right)$ and $\bar{h}^{\prime}\left(b^{\prime}\right)=\bar{f}(b)=b$. Let 


$$
\begin{aligned}
& h_{b}=h \mid p^{-1}(b): p^{-1}(b) \longrightarrow p^{\prime-1}\left(b^{\prime}\right), \\
& h_{b^{\prime}}^{\prime}=h^{\prime} \mid p^{\prime-1}\left(b^{\prime}\right): p^{\prime-1}\left(b^{\prime}\right) \longrightarrow p^{-1}(b) .
\end{aligned}
$$

Then $f_{b}=h_{b^{\prime}}^{\prime} \circ h_{b}$ and $g_{b^{\prime}}=h_{b} \circ h_{b^{\prime}}^{\prime}$. Let $K$ be the kernel of $\left(i_{b}\right)_{\pi}$ : $\pi_{1}\left(p^{-1}(b)\right) \rightarrow \pi_{1}(E)$ and let $K^{\prime}$ be the kernel of $\left(i_{b^{\prime}}\right)_{\pi}: \pi_{1}\left(p^{\prime-1}\left(b^{\prime}\right)\right) \rightarrow \pi_{1}\left(E^{\prime}\right)$. The equations $i_{b^{\prime}} \circ h_{b}=h \circ i_{b}$ and $i_{b} \circ h_{b^{\prime}}^{\prime}=h^{\prime} \circ i_{b^{\prime}}$ imply $\left(h_{b}\right)_{\pi}(K) \subset K^{\prime}$ and $\left(h_{b^{\prime}}^{\prime}\right)_{\pi}\left(K^{\prime}\right) \subset K$. By Corollary 1.5, we get the bijection $\left(h_{b}\right)_{r}$ : $\nabla_{K}\left(f_{b}\right) \rightarrow \nabla_{K^{\prime}}\left(g_{b^{\prime}}\right)$ which preserves index. By Lemma 1.4, the diagram

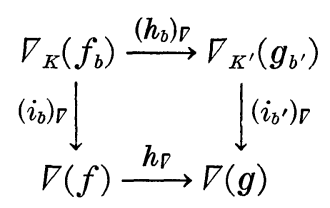

is commutative. It follows that $j(\boldsymbol{F})=j\left(\boldsymbol{F}^{\prime}\right)$ and $k(\boldsymbol{F})=k\left(\boldsymbol{F}^{\prime}\right)$.

A space $X$ dominates a space $Y$ by maps $Y \stackrel{\eta}{\rightarrow} X \stackrel{\xi}{\rightarrow} Y$ if $\xi \circ \eta \simeq$ id: $Y \rightarrow Y$. We say that a fiber space $\left(E^{\prime}, p^{\prime}, B^{\prime}\right)$ fiber dominates a fiber space $(E, p, B)$ by fiber maps $E \stackrel{\eta}{\rightarrow} E^{\prime} \stackrel{\xi}{\rightarrow} E$ if $\xi \circ \eta$ is fiber homotopic to the identity of $E$.

The following Lemmas 4.4, 4.5 and 4.6 are due to Boju Jiang.

Lemma 4.4. Let $(E, p, B)$ be a fiber space with $E, B$ and all fibers compact connected ANR's, let $B^{\prime}$ be a compact connected ANR, and let $\bar{\xi}: B^{\prime} \rightarrow B$ be a map. Let $\left(E^{\prime}, p^{\prime}, B^{\prime}\right)$ be the fiber space induced from $p$ by $\bar{\xi}$. Then $E^{\prime}$ and all the fibers of $\left(E^{\prime}, p^{\prime}, B^{\prime}\right)$ are compact connected ANR's.

Proof. By definition (cf. [10], p. 98), $E^{\prime}$ is the closed subspace $\left\{\left(b^{\prime}, e\right) \mid \bar{\xi}\left(b^{\prime}\right)=p(e)\right\}$ of $B^{\prime} \times E$. For any $b^{\prime} \in B^{\prime}$, the fiber $p^{\prime-1}\left(b^{\prime}\right)$ is $\left\{b^{\prime}\right\} \times p^{-1}\left(\bar{\xi}\left(b^{\prime}\right)\right)$. Since $p^{-1}\left(\bar{\xi}\left(b^{\prime}\right)\right)$ is a compact connected ANR, so is $p^{\prime-1}\left(b^{\prime}\right)$. From the connectedness of $B^{\prime}$ and all fibers of $\left(E^{\prime}, p^{\prime}, B^{\prime}\right)$ we can easily see that $E^{\prime}$ is also connected.

It remains to show that $E^{\prime}$ is a compact ANR. Since $B^{\prime}$ and $E$ are compact ANR's, so is $B^{\prime} \times E$. The closed subspace $E^{\prime}$ of $B^{\prime} \times E$ is certainly compact. To show $E^{\prime}$ is an ANR, we need only prove that $E^{\prime}$ is a neighborhood retract of $B^{\prime} \times E$.

According to the uniform local contractibility of the ANR $B$ (cf. [3], p. 39), there exists $\delta>0$ and for every pair $\left(b_{0}, b_{1}\right)$ of points of $B$ with $d\left(b_{0}, b_{1}\right)<\delta$ there exists a path $\gamma\left(b_{0}, b_{1}\right)$ from $b_{0}$ to $b_{1}$ such that $\gamma\left(b_{0}, b_{1}\right)$ depends continuously on $b_{0}$ and $b_{1}$, and $\gamma\left(b_{0}, b_{1}\right)$ is the constant path if $b_{0}=b_{1}$. Now define

$$
r:\left\{\left(b^{\prime}, e\right) \mid d\left(\bar{\xi}\left(b^{\prime}\right), p(e)\right)<\delta\right\} \longrightarrow E^{\prime}
$$


by

$$
r\left(b^{\prime}, e\right)=\left(b^{\prime}, \tau_{\gamma\left(p(e), \bar{\xi}\left(b^{\prime}\right)\right)}(e)\right) .
$$

It is easy to check that $r$ is a retraction and $\left\{\left(b^{\prime}, e\right) \mid d\left(\bar{\xi}\left(b^{\prime}\right), p(e)\right)<\delta\right\}$ is open in $B^{\prime} \times E$. Hence $E^{\prime}$ is a neighborhood retract of $B^{\prime} \times E$, and therefore it is an ANR.

Lemma 4.5. Let $(E, p, B)$ be a fiber space with $E, B$ and all fibers compact connected ANR's. Then $(E, p, B)$ is fiber dominated by another such fiber space with polyhedral base space.

Proof. There is a compact connected polyhedron $B^{\prime}$ which dominates $B$ by $B \stackrel{\tilde{\eta}}{\rightarrow} B^{\prime} \stackrel{\bar{\xi}}{\rightarrow} B$. Let $\left(E^{\prime}, p^{\prime}, B^{\prime}\right)$ be the fiber space induced by $\bar{\xi}$ from $p$. The map $\bar{\xi}$ lifts to a fiber map $\xi: E^{\prime} \rightarrow E$. Lemma 4.4 shows that $E^{\prime}$ and all fibers of $\left(E^{\prime}, p^{\prime}, B^{\prime}\right)$ are also compact connected ANR's. It remains to show $\left(E^{\prime}, p^{\prime}, B^{\prime}\right)$ fiber dominates $(E, p, B)$.

Let $\left(E^{\prime \prime}, p^{\prime \prime}, B\right)$ be the fiber space induced by $\bar{\eta}$ from $p^{\prime}$ and lift $\bar{\eta}$ to $\eta: E^{\prime \prime} \rightarrow E^{\prime}$. Then $\left(E^{\prime \prime}, p^{\prime \prime}, B\right)$ is the fiber space induced by $\bar{\xi} \circ \bar{\eta}$ from $p$. But $\bar{\xi} \circ \bar{\eta} \simeq$ id, so (cf. [10], p. 102) $\xi \circ \eta: E^{\prime \prime} \rightarrow E$ is a fiber homotopy equivalence with a fiber homotopy inverse $\zeta: E \rightarrow$ $E^{\prime \prime}$. Now $E \stackrel{\eta^{\circ \zeta}}{\rightarrow} E^{\prime} \stackrel{\xi}{\rightarrow} E$ is a domination of $E$ by $E^{\prime}$.

Lemma 4.6. Let $(E, p, B)$ be a fiber space with $E, B$ and all fibers compact connected ANR's, and let $f: E \rightarrow E$ be a fiber map. Suppose $B$ is a polyhedron, and $\bar{f}: B \rightarrow B$ has an isolated fixed point $b$ which lies in some maximal open simplex $\sigma \subset B$. Let $A \subset \Phi\left(f_{b}\right)$ be both closed and open in $\Phi\left(f_{b}\right)$, then

$$
i_{f}(A)=i_{\bar{f}}(b) \cdot i_{f_{b}}(A) \text {. }
$$

Proof. For any two points $b_{1}, b_{2} \in \sigma$, let $\tau\left(b_{1}, b_{2}\right): p^{-1}\left(b_{1}\right) \rightarrow p^{-1}\left(b_{2}\right)$ stand for the translation determined by the linear path from $b_{1}$ to $b_{2}$. Note that $\tau\left(b_{1}, b_{2}\right)=$ id, if $b_{1}=b_{2}$.

Pick a Euclidean neighborhood $U$ of $b$ such that $\bar{U} \cup \bar{f}(\bar{U}) \subset \sigma$ and $b$ is the only fixed point of $\bar{f}$ on $\bar{U}$. Define maps

$$
\iota: \bar{\sigma} \times p^{-1}(b) \longrightarrow E \text { by } \iota\left(b^{\prime}, y\right)=\tau\left(b, b^{\prime}\right)(y),
$$

and

$$
\varphi: p^{-1}(\bar{U}) \longrightarrow \bar{\sigma} \times p^{-1}(b) \text { by } \varphi(e)=(\bar{f} \circ p(e), f \circ \tau(p(e), b)(e)) .
$$

There are homotopies $\left\{h_{t}\right\}: \bullet \circ \varnothing \simeq f: p^{-1}(\bar{U}) \rightarrow E$ and $\left\{k_{t}\right\}: \bar{f} \times f_{b} \simeq \varnothing \circ ८$ : $\bar{U} \times p^{-1}(b) \rightarrow \sigma$, defined by 


$$
h_{t}(e)=\tau(\bar{f}((1-t) b+t p(e)), \bar{f} \circ p(e)) \circ f \circ \tau(p(e),(1-t) b+t p(e))(e)
$$

and

$$
k_{t}\left(b^{\prime}, y\right)=\left(\bar{f}\left(b^{\prime}\right), f \circ \tau\left((1-t) b+t b^{\prime}, b\right) \circ \tau\left(b,(1-t) b+t b^{\prime}\right)(y)\right) .
$$

Then $\Phi\left(h_{t}\right)=\Phi\left(f_{b}\right)$ and $\Phi\left(k_{t}\right)=b \times \Phi\left(f_{b}\right)$, for all $t$.

Now take a neighborhood $W \subset p^{-1}(U)$ of $A$ in $E$, such that $\bar{W} \cap \Phi(f)=A$. Let $W_{b}=W \cap p^{-1}(b)$. Then by definition we have $i_{f}(A)=i(f, W)$, and $i_{f_{b}}(A)=i\left(f_{b}, W_{b}\right)$, where $i$ denotes the fixed index of a map on an open set. We have

$$
\begin{aligned}
i(f, W) & =i(\iota \circ \varphi, w) & & \text { (homotopy invariance of the index) } \\
& =i\left(\varphi \circ \iota, \iota^{-1}(w)\right) & & \text { (commutativity of the index) } \\
& =i\left(\varphi \circ \iota, U \times W_{b}\right) & & \text { (both sets contain the same fixed points) } \\
& =i\left(\bar{f} \times f_{b}, U \times W_{b}\right) & & \text { (homotopy invariance of the index) } \\
& =i(\bar{f}, U) \cdot i\left(f_{b}, W_{b}\right) . & &
\end{aligned}
$$

The last equation is by Exercise V. 3 of [3].

The conclusion of the lemma now follows, because $i_{\bar{f}}(b)=$ $i(\bar{f}, U)$.

Proof of Theorem 4.1. By Lemma 4.5, we get a fiber space $\left(E^{\prime}, p^{\prime}, B^{\prime}\right)$ with $E^{\prime}$ and all fibers compact connected ANR's, and $B^{\prime}$ a compact connected polyhedron, which fiber dominates $(E, p, B)$ by fiber maps $E \stackrel{\eta}{\rightarrow} E^{\prime} \stackrel{\xi}{\rightarrow} E$. We define $f_{1}=\xi \circ \eta \circ f: E \rightarrow E$ and $f^{\prime}=\eta \circ$ $f \circ \xi: E^{\prime} \rightarrow E^{\prime}$. By Hopf's Approximation Theorem (cf. [3], p. 118), $\bar{f}^{\prime}$ is homotopic to a map $\bar{f}^{\prime \prime}: B^{\prime} \rightarrow B^{\prime}$ such that $\bar{f}^{\prime \prime}$ has only a finite number of fixed points each lying in a maximal simplex of some triangulation of $B^{\prime}$. By the homotopy lifting property of $\left(E^{\prime}, p^{\prime}, B^{\prime}\right)$, $\bar{f}^{\prime \prime}$ lifts to a fiber map $f^{\prime \prime}: E^{\prime} \rightarrow E^{\prime}$ which is fiber homotopic to $f^{\prime}$. The proof is in four steps.

Step 1. The theorem holds for $f^{\prime \prime}$.

Let $\boldsymbol{F} \in \Phi^{\prime}\left(f^{\prime \prime}\right)$, and $p_{\sigma}^{\prime}(\boldsymbol{F})=\left\{b_{1}^{\prime}, \cdots, b_{s}^{\prime}\right\}$. Then

$$
\begin{array}{rlr}
i_{f^{\prime \prime}}(\boldsymbol{F}) & =\sum_{i=1}^{s} i_{f^{\prime \prime}}\left(\boldsymbol{F} \cap p^{\prime-1}\left(b_{i}^{\prime}\right)\right) \\
& =\sum_{i=1}^{s} i_{\bar{f}^{\prime \prime}}\left(b_{i}^{\prime}\right) \cdot i_{f^{\prime \prime} \mid p^{\prime-1}\left(b_{i}^{\prime}\right)}\left(\boldsymbol{F} \cap p^{\prime-1}\left(b_{i}^{\prime}\right)\right) & (\text { Lemma 4.6) } \\
& =j(\boldsymbol{F}) \cdot k(\boldsymbol{F}) \cdot \sum_{i=1}^{s} i_{\bar{f}^{\prime \prime}}\left(\boldsymbol{b}_{i}^{\prime}\right) & \text { (Definition 3.5) } \\
& =j(\boldsymbol{F}) \cdot k(\boldsymbol{F}) \cdot i_{\bar{f}^{\prime \prime}}\left(p_{\bar{v}}^{\prime}(\boldsymbol{F})\right) . &
\end{array}
$$

It follows that $i_{f^{\prime \prime}}(\boldsymbol{F}) \neq 0 \Leftrightarrow j(\boldsymbol{F}) \neq 0$ and $i_{\bar{f}^{\prime \prime}}\left(p_{\nabla}^{\prime}(\boldsymbol{F})\right) \neq 0$. 
Step 2. The theorem holds for $f^{\prime}$.

Let $F$ be a fiber homotopy from $f^{\prime}$ to $f^{\prime \prime}$. For each $\boldsymbol{F} \in \Phi^{\prime}\left(f^{\prime}\right)$, let $\boldsymbol{F}^{\prime}=\mu_{F}(\boldsymbol{F})$. If $i_{f^{\prime}}(\boldsymbol{F}) \neq 0$, then $i_{f^{\prime \prime}}\left(\boldsymbol{F}^{\prime}\right)=i_{f^{\prime}}(\boldsymbol{F}) \neq 0$. By Step 1, $j\left(\boldsymbol{F}^{\prime}\right) \neq 0$ and $i_{\bar{f}^{\prime \prime}}\left(p_{\nabla}^{\prime}\left(\boldsymbol{F}^{\prime}\right)\right) \neq 0$. Then, by Lemma 4.2, $j(\boldsymbol{F}) \neq 0$ and $i_{\bar{f}^{\prime}}\left(p_{\bar{\nabla}}^{\prime}(\boldsymbol{F})\right) \neq 0$. Conversely, if $j(\boldsymbol{F}) \neq 0$ and $i_{\bar{f}^{\prime}}\left(p_{\bar{\nabla}}^{\prime}(\boldsymbol{F})\right) \neq 0$, then by Lemma 4.2 again, $j\left(\boldsymbol{F}^{\prime}\right) \neq 0$ and $i_{\bar{f}^{\prime \prime}}\left(p^{\prime}\left(\boldsymbol{F}^{\prime}\right)\right) \neq 0$. By Step 1, $i_{f^{\prime}}(\boldsymbol{F})=$ $i_{f^{\prime \prime}}\left(\boldsymbol{F}^{\prime}\right) \neq 0$.

Step 3. The theorem holds for $f_{1}$.

To prove it, one can adopt the method of Step 2, using Lemma 4.3 instead of 4.2 .

Step 4. The theorem holds for $f$.

The proof is similar to Step 2 .

5. The Nielsen number of a fiber map $f$. Let $\overline{\boldsymbol{F}}_{1}, \cdots, \overline{\boldsymbol{F}}_{n}$ be the essential fixed point classes of $\bar{f}$, where $n=N(\bar{f})$. By Theorem 4.1, if $\boldsymbol{F} \in \nabla(f)$ is essential, then $p_{\nabla}(f)$ is also essential, so for some $i, p_{\nabla}(\boldsymbol{F})=\widetilde{\boldsymbol{F}}_{i}$. Let

$$
c_{i}=\sharp\left\{\boldsymbol{F} \in \nabla(f) \mid i_{f}(\boldsymbol{F}) \neq 0, p_{\nabla}(\boldsymbol{F})=\overline{\boldsymbol{F}}_{i}\right\}, \quad 1 \leqq i \leqq n,
$$

then

$$
N(f)=\sum_{i=1}^{n} C_{i} .
$$

Thus to calculate $N(f)$, we need only calculate the $C_{i}$.

Definition 5.1. Let $X$ be a space and let $h: X \rightarrow X$ be a map. If $x \in \Phi(h)$, then $h_{\pi}: \pi_{1}(X, x) \rightarrow \pi_{1}(X, x)$. We define

$$
\operatorname{Fix}\left(h_{\pi}\right)_{x}=\left\{\alpha \in \pi_{1}(X, x) \mid h_{\pi}(\alpha)=\alpha\right\},
$$

which is a subgroup of $\pi_{1}(X, x)$.

Let $b \in \Phi(\bar{f})$. Then we have $\operatorname{Fix}\left(\bar{f}_{\pi}\right)_{b}$. If $\alpha=\langle\bar{w}\rangle \in \operatorname{Fix}\left(\bar{f}_{\pi}\right)_{b}$, then $\bar{w} \simeq \bar{f} \circ \bar{w}$. Lemma 2.7 permits us to define $T_{\alpha}=T_{\bar{w}}: \nabla_{K}\left(f_{b}\right) \rightarrow$ $\nabla_{K}\left(f_{b}\right)$. If $\alpha, \alpha^{\prime} \in \operatorname{Fix}\left(\bar{f}_{\pi}\right)_{b}$, then by Lemma $2.8 T_{\alpha \alpha^{\prime}}=T_{\alpha^{\prime}} \circ T_{\alpha}$. Thus Fix $\left(\bar{f}_{\pi}\right)_{b}$ is a transformation group acting on $\nabla_{K}\left(f_{b}\right)$ on the right. Since $T_{\alpha}$ preserves index, all elements of an orbit of Fix $\left(\bar{f}_{\pi}\right)_{b}$ have the same index. An orbit is called essential if it consists of essential elements.

LeMma 5.2. For any $b \in \Phi(\bar{f})$ and any $\boldsymbol{F} \in \operatorname{Im}\left(\left(i_{b}\right)_{\nabla}\right)$, the set $\left(i_{b}\right)_{\bar{\nu}}^{-1}(\boldsymbol{F})$ is exactly an orbit of Fix $\left(\bar{f}_{\pi}\right)_{b}$ acting on $\nabla_{K}\left(f_{b}\right)$. Moreover, if $b \in \overline{\boldsymbol{F}}_{i}$, then the number of essential orbits is equal to $C_{i}$. 
Proof. By Theorem 3.1, two classes $\boldsymbol{F}_{0}, \boldsymbol{F}_{1} \in \nabla_{K}\left(f_{b}\right)$ are in the same orbit if and only if $\left(i_{b}\right)_{\nabla}\left(\boldsymbol{F}_{0}\right)=\left(i_{b}\right)_{\nabla}\left(\boldsymbol{F}_{1}\right)$. Then the first conclusion follows.

Now let $b \in \overline{\boldsymbol{F}}_{i}$. By Theorem 4.1, $\boldsymbol{F} \in \operatorname{Im}\left(\left(i_{b}\right)_{r}\right)$ is essential if and only if $\left(i_{b}\right)_{\nabla}^{-1}(\boldsymbol{F})$ is an essential orbit. Then $\left(i_{b}\right)_{\nu}$ induces an injection from the collection of the essential orbits to $\left\{\boldsymbol{F} \in \nabla(f) \mid i_{f}(\boldsymbol{F}) \neq 0\right.$, $\left.p_{\nabla}(\boldsymbol{F})=\overline{\boldsymbol{F}}_{i}\right\}$. It is also surjective, because any $\boldsymbol{F} \in\left\{\boldsymbol{F} \in \nabla(f) \mid i_{f}(\boldsymbol{F}) \neq 0\right.$, $\left.p_{\nabla}(\boldsymbol{F})=\overline{\boldsymbol{F}}_{i}\right\}$ contains $k(\boldsymbol{F})$ essential elements of $\nabla_{K}\left(f_{b}\right)$. Hence we get the second conclusion.

LEMMA 5.3. Let $b \in \Phi(\bar{f}), \quad \boldsymbol{F}_{0} \in \Phi_{K}^{\prime}\left(f_{b}\right)$ and $\alpha \in \operatorname{Fix}\left(\bar{f}_{\pi}\right)_{b}$. Then $T_{\alpha}\left(\boldsymbol{F}_{0}\right)=\boldsymbol{F}_{0}$ if and only if for any $x \in \boldsymbol{F}_{0}, \alpha \in p_{\pi}\left(\operatorname{Fix}\left(f_{\pi}\right)_{x}\right)$.

Proof. "If." We take $x \in \boldsymbol{F}_{0}$. Let $\langle v\rangle \in \operatorname{Fix}\left(f_{\pi}\right)_{x}$ make $p_{\pi}(\langle v\rangle)=$ $\alpha$. Then $v \simeq f \circ v$ and $\bar{w}=p \circ v \in \alpha$, so $T_{\alpha}=T_{\bar{w}}$. Take a reference pair $\left(x, e_{x}\right)$ for $f_{b}\left(e_{x}\right.$ denotes the constant path at $\left.x\right)$. Then the representation of $\boldsymbol{F}_{0}$ in $\left(x, e_{x}\right)$ is $\left[\left\langle e_{x}\right\rangle_{k}\right]$ (Lemma 1.1). Let $\left(x^{\prime}, r^{\prime}\right)$ be the induced pair for $f_{b}$ from $\left(x, e_{x}\right)$ and $\bar{w}$, then the representation of $T_{\alpha}\left(\boldsymbol{F}_{0}\right)$ in $\left(x^{\prime}, r^{\prime}\right)$ is $\left[\left\langle e_{x^{\prime}}\right\rangle_{k}\right]$ (by (4)). Let $w=\lambda(x, \bar{w})$, which is a path from $x$ to $x^{\prime}$. Since $\left\langle p \circ\left(w^{-1} v\right)\right\rangle$ is the unit of $\pi_{1}(B, b)$, by Lemma 2.6, there is a path $\ell$ in $p^{-1}(b)$ from $x^{\prime}$ to $x$ such that $\ell \simeq$ $w^{-1} v$ in $E$. Then by (1), the representation of $T_{\alpha}\left(\boldsymbol{F}_{0}\right)$ in $\left(x, e_{x}\right)$ is $\left[\left\langle\ell^{-1} r^{\prime}(f \circ \ell)\right\rangle_{K}\right]$. By (5), in $E$

$$
\begin{aligned}
\ell^{-1} r^{\prime}(f \circ \ell) & \simeq v^{-1} w w^{-1}(f \circ w)\left(f \circ w^{-1}\right)(f \circ v) \\
& \simeq v^{-1}(f \circ v) \simeq e_{x},
\end{aligned}
$$

so $\left\langle\ell^{-1} \boldsymbol{r}^{\prime}(f \circ \ell)\right\rangle_{K}=\left\langle e_{x}\right\rangle_{K}$ and $T_{\alpha}\left(\boldsymbol{F}_{0}\right)=\boldsymbol{F}_{0}$.

"Only if." For any $x \in \boldsymbol{F}_{0}$, we take a reference pair $\left(x, e_{x}\right)$ for $f_{b}$. Let $\bar{w} \in \alpha$ and $\left(x^{\prime}, r^{\prime}\right)$ be the induced pair from $\left(x, e_{x}\right)$ and $\bar{w}$. Then the representation of $\boldsymbol{F}_{0}$ in $\left(x, e_{x}\right)$ is $\left[\left\langle e_{x}\right\rangle_{K}\right]$, and the representation of $\boldsymbol{T}_{\alpha}\left(\boldsymbol{F}_{0}\right)$ in $\left(x^{\prime}, \boldsymbol{r}^{\prime}\right)$ is $\left[\left\langle e_{x^{\prime}}\right\rangle_{K}\right]$ (by (4)). Since $T_{\alpha}\left(\boldsymbol{F}_{0}\right)=\boldsymbol{F}_{0}$, by Lemma 1.1, we can find a path $c$ in $p^{-1}(b)$ from $x$ to $x^{\prime}$ such that $\left\langle c^{-1}(f \circ c) r^{\prime-1}\right\rangle_{K}=\left\langle e_{x^{\prime}}\right\rangle_{K}$. Then in $E$

$$
c^{-1}(f \circ c) r^{\prime-1} \simeq e_{x^{\prime}} \text {. }
$$

Let $w=\lambda(x, \bar{w})$. Then $r^{\prime} \simeq w^{-1}(f \circ w)$ in $E$ (by (5)), so in $E$

$$
w c^{-1} \simeq f \circ\left(w c^{-1}\right) \text {. }
$$

Thus $\left\langle w c^{-1}\right\rangle \in \operatorname{Fix}\left(f_{\pi}\right)_{x}$ and $p_{\pi}\left(\left\langle w c^{-1}\right\rangle\right)=\langle\bar{w}\rangle=\alpha$, so $\alpha \in p_{\pi}\left(\operatorname{Fix}\left(f_{\pi}\right)_{x}\right)$.

For any $x \in \Phi(f)$, one can easily check that $p_{\pi}\left(\operatorname{Fix}\left(f_{\pi}\right)_{x}\right) \subset \operatorname{Fix}\left(\bar{f}_{\pi}\right)_{b}$, where $b=p(x)$. Lemma 5.3 shows that the isotropy group of $F_{0}$ is $p_{\pi}\left(\operatorname{Fix}\left(f_{\pi}\right)_{x}\right)$, for any $x \in \boldsymbol{F}_{0}$. 
CoROLLARY 5.4. Let $b \in \Phi(\bar{f})$ and $\boldsymbol{F}_{0} \in \Phi_{K}^{\prime}\left(f_{b}\right)$, then the length of the orbit of $\boldsymbol{F}_{0}$ is equal to the index of $p_{\pi}\left(\operatorname{Fix}\left(f_{\pi}\right)_{x}\right)$ in Fix $\left(\bar{f}_{\pi}\right)_{b}$, written $\left[\mathrm{Fix}\left(\bar{f}_{\pi}\right)_{b}: p_{\pi}\left(\mathrm{Fix}\left(f_{\pi}\right)_{x}\right)\right]$, where $x \in \boldsymbol{F}_{0}$.

We now discuss the product formula of the Nielsen number of a fiber map. Its original form is

$$
N(f)=N(\bar{f}) \cdot N\left(f_{b}\right) .
$$

It does not always hold. Now we discuss the conditions which imply (7), and improve (7).

THEOREM 5.5. The formula (7) holds, if one of the following conditions is satisfied:

(i ) $N(\bar{f})=0$,

(ii) $N\left(f_{b}\right)=0$, for any $b \in \overline{\boldsymbol{F}}_{i}, i=1, \cdots, n$,

(iii) $N\left(f_{b}\right)=1$, for any $b \in \overline{\boldsymbol{F}}_{i}, i=1, \cdots, n$.

Proof. According to Theorem 4.1, $N(\bar{f})=0$ implies $N(f)=0$. Thus if (i) is satisfied, then $N(f)=0$ and (7) holds. If (ii) or (iii) is satisfied, then $N\left(f_{b}\right)=N_{K}\left(f_{b}\right)=C_{i}$ for all $b \in \overline{\boldsymbol{F}}_{i}$, and (7) follows from (6).

In general, $N\left(f_{b}\right)$ depends on $b$. But if $(E, p, B)$ is orientable in the sense that for any loop $\bar{w}$ in $B$ based at $b$ the translation $\tau_{\bar{w}} \simeq \mathrm{id}: p^{-1}(b) \rightarrow p^{-1}(b)$, then both $N\left(f_{b}\right)$ and $N_{K}\left(f_{b}\right)$ are independent of the choice of $b \in \Phi(\bar{f})$.

THEOREM 5.6. Let $(E, p, B)$ be orientable, and $f: E \rightarrow E$ be a fiber map such that $N(f) \neq 0$. Then (7) holds if and only if

(a) $N_{K}\left(f_{b}\right)=N\left(f_{b}\right)$; and

(b) for any $x \in \Phi(f)$ belonging to an essential class, $p_{\pi}\left(\operatorname{Fix}\left(f_{\pi}\right)_{x}\right)=$ Fix $\left(\bar{f}_{\pi}\right)_{b}$, where $b=p(x)$.

Proof. By Corollary 5.4, (b) is equivalent to the condition that for any $b \in \overline{\boldsymbol{F}}_{i}$, the length of every essential orbit of Fix $\left(\bar{f}_{\pi}\right)_{b}$ is equal to 1 , for $i=1, \cdots, n$. Then by Lemma 5.2 , it is equivalent to $C_{i}=N_{K}\left(f_{b}\right), i=1, \cdots, n$. Then from (6), (b) is a necessary and sufficient condition for the formula

$$
N(f)=N(\bar{f}) \cdot N_{K}\left(f_{b}\right)
$$

to hold. The conclusion of the theorem now follows.

Theorems 5.5 and 5.6 show that, for an orientable fiber space, 
when conditions (a) and (b) are satisfied, the formula (7) always holds.

Corollary 5.7 (Fadell [4]). Let $(E, p, B)$ be orientable, and let $f: E \rightarrow E$ be a fiber map. Suppose $(E, p, B)$ admits a natural fiber splitting with respect to $f$. That is, for each $x \in E$ the following conditions are satisfied:

(i) the sequence

$$
0 \longrightarrow \pi_{1}\left(p^{-1}(b), x\right) \stackrel{\left(i_{b}\right)_{\pi}}{\longrightarrow} \pi_{1}(E, x) \stackrel{p_{\pi}}{\longrightarrow} \pi_{1}(B, b) \longrightarrow 0
$$

is exact, where $b=p(x)$;

(ii) $p_{\pi}$ admits a right inverse $\sigma$ such that $\operatorname{Im} \sigma$ is normal in $\pi_{1}(E)$ and $f_{\pi}(\operatorname{Im} \sigma) \subset w_{*}(\operatorname{Im} \sigma)$ for any path $w$ in $E$ from $x$ to $f(x)$ (in fact, $w_{*}(\operatorname{Im} \sigma)$ is independent of the choice of $w$ ). Then (7) holds.

Proof. Condition (i) implies $K$ is trivial and (a) of Theorem 5.6 is satisfied.

For any $x \in \Phi(f)$, let $b=p(x)$. For any $\alpha \in \operatorname{Fix}\left(\bar{f}_{\pi}\right)_{b}$, let $\beta=\sigma(\alpha)$. By (ii), we have $f_{\pi}(\beta) \in \operatorname{Im} \sigma$. Let $f_{\pi}(\beta)=\sigma\left(\alpha^{\prime}\right)$, then

$$
\alpha^{\prime}=p_{\pi} \circ f_{\pi}(\beta)=\bar{f}_{\pi} \circ p_{\pi}(\beta)=\bar{f}_{\pi}(\alpha)=\alpha,
$$

so $f_{\pi}(\beta)=\sigma(\alpha)=\beta$. Thus $\alpha \in p_{\pi}\left(\operatorname{Fix}\left(f_{\pi}\right)_{x}\right)$. Hence (b) of Theorem 5.6 is also satisfied, and (7) holds.

CoRollary 5.8 (Fadell [4]). Let $(E, p, B)$ be orientable and let $f: E \rightarrow E$ be a fiber map such that for any $b \in \Phi(\bar{f})$ which belongs to an essential class and for any $x \in p^{-1}(b)$

$$
\left(i_{b}\right)_{\pi}: \pi_{1}\left(p^{-1}(b), x\right) \longrightarrow \pi_{1}(E, x)
$$

is injective, and Fix $\left(\bar{f}_{\pi}\right)_{b}$ is trivial. Then (7) holds.

Proof. Since $\left(i_{b}\right)_{\pi}$ is injective, then (a) of Theorem 5.6 is satisfied. Since Fix $\left(\bar{f}_{\pi}\right)_{b}$ is trivial, then (b) of Theorem 5.6 is satisfied.

EXAmple 1. Let $(E, p, B)$ be orientable and $B=T^{n}$, the $n$-torus, then (7) always holds for any fiber map $f: E \rightarrow E$.

Proof. Since $\pi_{2}\left(T^{n}\right)=0$, then $\left(i_{b}\right)_{\pi}: \pi_{1}\left(p^{-1}(b)\right) \rightarrow \pi_{1}(E)$ is injective. It follows that (a) of Theorem 5.6 is satisfied.

The group $\pi_{1}\left(T^{n}\right)$ is free abelian with $n$ generators. When the generators are given, then each element $\alpha \in \pi_{1}\left(T^{n}\right)$ can be denoted by $\left(k_{1}, \cdots, k_{n}\right)$, where the $k_{i}$ are integers, and $\bar{f}_{\pi}: \pi_{1}\left(T^{n}\right) \rightarrow \pi_{1}\left(T^{n}\right)$ can be represented by an $n \times n$ matrix $A$ with integer elements: 


$$
\bar{f}_{\pi}(\alpha)=A \alpha .
$$

By [1], $N(\bar{f})=|\operatorname{det}(A-I)|$ where $I$ denotes the identity matrix. If $\bar{f}_{\pi}$ fixed only the unit, then by Corollary $5.8,(7)$ holds. Otherwise, there is an $\alpha \in \pi_{1}(B)$ such that $\alpha$ is not the unit and $\bar{f}_{\pi}(\alpha)=\alpha$. It follows that $(A-I) \alpha=0$, and thus $N(\bar{f})=|\operatorname{det}(A-I)|=0$. By Theorem 5.5, (7) still holds.

EXAMPle 2. Let $S_{1}^{1}$ denote the unit circle in the complex plane $C$, that is $S_{1}^{1}=\{z \in C|| z \mid=1\}$, and let $S_{2}^{1}=\{z \in C|| z-2 \mid=1\}$. Let $B=S_{1}^{1} \cup S_{2}^{1}, E=B \times S_{1}^{1}$ and $p: E \rightarrow B$ be the projection. Then $(E, p, B)$ is a trivial fiber space, so it is orientable and (a) of Theorem 5.6 is satisfied. We define a fiber map $f: E \rightarrow E$ by

$$
f(b, y)= \begin{cases}\left(b^{2}, b^{\zeta} y^{m}\right), & \text { if } b \in S_{1}^{1}, \\ \left(b, y^{m}\right), & \text { if } b \in S_{2}^{1},\end{cases}
$$

where $\ell, m$ are integers. Then $\Phi(\bar{f})=S_{2}^{1}$ and $N(\bar{f})=1$. The group $\pi_{1}(B)$ is free with two generators. For any $b \in S_{2}^{1}$, let $\bar{c}$ be the loop in $B$ based at $b$ which goes around $S_{2}^{1}$ once. Then Fix $\left(\bar{f}_{\pi}\right)_{b}$ is exactly the free cyclic group generated by $\langle\bar{c}\rangle$. Let $(b, y) \in \Phi(f)$, and let a path $\left(\bar{c}, e_{y}\right)$ be defined by $\left(\bar{c}, e_{y}\right)(t)=(\bar{c}(t), y)$. Then $\left(\bar{c}, e_{y}\right)$ is a loop in $E$ based at $(b, y)$ and $\left\langle\left(\bar{c}, e_{y}\right)\right\rangle \in \operatorname{Fix}\left(f_{\pi}\right)_{(b, y)}$. But $p_{\pi}\left(\left\langle\left(\bar{c}, e_{y}\right)\right\rangle\right)=$ $\langle\bar{c}\rangle$, so $\operatorname{Fix}\left(\bar{f}_{\pi}\right)_{b}=p_{\pi}\left(\operatorname{Fix}\left(f_{\pi}\right)_{(b, y)}\right)$. Thus (b) of Theorem 5.6 is satisfied and (7) holds for $f$.

Note that Example 2 does not satisfy the conditions of Corollary 5.8 , and if $(2-m) \nmid \ell$ it does not satisfy the conditions of Corollary 5.7 .

When $(E, p, B)$ is orientable, $C_{i} \leqq N_{K}\left(f_{b}\right) \leqq N\left(f_{b}\right)$, so from (6),

$$
N(f) \leqq N(\bar{f}) N_{K}\left(f_{b}\right) \leqq N(\bar{f}) \cdot N\left(f_{b}\right) .
$$

If $N(f) \neq 0$, define rational numbers

$$
\begin{aligned}
P_{K}(f) & =\left(N(\bar{f}) \cdot N_{K}\left(f_{b}\right)\right) / N(f), \\
P(f) & =\left(N(\bar{f}) \cdot N\left(f_{b}\right)\right) / N(f) .
\end{aligned}
$$

Note that $P(f) \geqq P_{K}(f) \geqq 1$. We must calculate $P_{K}(f)$ and $P(f)$.

Theorem 5.9. Let $(E, p, B)$ be orientable and let $f: E \rightarrow E$ be eventually commutative, that is, $\left(f^{q}\right)_{\pi}\left(\pi_{1}(E)\right)$ is abelian for some positive integer $q$ (cf. [7], p. 61). If $N(f) \neq 0$, then for any $x \in \Phi(f)$ and $b=p(x)$,

$$
P_{K}(f)=\left[\operatorname{Fix}\left(\bar{f}_{\pi}\right)_{b}: p_{\pi}\left(\operatorname{Fix}\left(f_{\pi}\right)_{x}\right)\right],
$$


and $P_{K}(f)$ is a factor of $N_{K}\left(f_{b}\right)$.

Proof. First, we prove that $\left[\mathrm{Fix}\left(\bar{f}_{\pi}\right)_{b}: p_{\pi}\left(\mathrm{Fix}\left(f_{\pi}\right)_{x}\right)\right]$ is independent of the choice of $x$ in $\Phi(f)$.

Let $q$ be a positive integer such that $\left(f^{q}\right)_{\pi}\left(\pi_{1}(E)\right)$ is abelian. For any $x, x^{\prime} \in \Phi(f)$, let $v$ be a path in $E$ from $x$ to $x^{\prime}$, and let $w=$ $f^{q} \circ v$. Set $\beta=\left\langle v\left(f \circ v^{-1}\right)\right\rangle \in \pi_{1}(E, x)$, then $\left(f^{q}\right)_{\pi}(\beta)=\left\langle w\left(f \circ w^{-1}\right)\right\rangle$. If $\alpha \in \operatorname{Fix}\left(f_{\pi}\right)_{x}$, then $\alpha=\left(f^{q}\right)_{\pi}(\alpha) \in \operatorname{Im}\left(\left(f^{q}\right)_{\pi}\right)$. Since $\operatorname{Im}\left(\left(f^{q}\right)_{\pi}\right)$ is abelian, then

$$
\begin{aligned}
\left\langle w\left(f \circ w^{-1}\right)\right\rangle \alpha & =\left(f^{q}\right)_{\pi}(\beta) \cdot\left(f^{q}\right)_{\pi}(\alpha)=\left(f^{q}\right)_{\pi}(\alpha) \cdot\left(f^{q}\right)_{\pi}(\beta) \\
& =\alpha\left\langle w\left(f \circ w^{-1}\right)\right\rangle .
\end{aligned}
$$

Then

$$
\begin{aligned}
f_{\pi}\left(w_{*}(\alpha)\right) & =(f \circ w)_{*}\left(f_{\pi}(\alpha)\right)=w_{*}\left(\left\langle w\left(f \circ w^{-1}\right)\right\rangle \alpha\left\langle w\left(f \circ w^{-1}\right)\right\rangle^{-1}\right) \\
& =w_{*}(\alpha) .
\end{aligned}
$$

Thus $w_{*}\left(\operatorname{Fix}\left(f_{\pi}\right)_{x}\right) \subset \operatorname{Fix}\left(f_{\pi}\right)_{x^{\prime}}$. Similarly we have $w_{*}^{-1}\left(\operatorname{Fix}\left(f_{\pi}\right)_{x^{\prime}}\right) \subset$ Fix $\left(f_{\pi}\right)_{x}$. Hence $w_{*}$ : Fix $\left(f_{\pi}\right)_{x} \rightarrow \operatorname{Fix}\left(f_{\pi}\right)_{x^{\prime}}$ is an isomorphism.

Since $p_{\pi}$ is surjective, $\left(f^{q}\right)_{\pi}\left(\pi_{1}(B)\right)=p_{\pi}\left(\left(f^{q}\right)_{\pi}\left(\pi_{1}(E)\right)\right)$ is also abelian. We can similarly prove that $\bar{w}_{*}$ : Fix $\left(\bar{f}_{\pi}\right)_{b} \rightarrow \operatorname{Fix}\left(\bar{f}_{\pi}\right)_{b^{\prime}}$ is an isomorphism, where $b=p(x), b^{\prime}=p\left(x^{\prime}\right)$ and $\bar{w}=p \circ w$. Obviously, the diagram

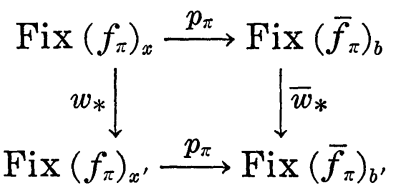

is commutative. It follows that

$$
\left[\operatorname{Fix}\left(\bar{f}_{\pi}\right)_{b}: p_{\pi}\left(\operatorname{Fix}\left(f_{\pi}\right)_{x}\right)\right]=\left[\operatorname{Fix}\left(\bar{f}_{\pi}\right)_{b^{\prime}}: p_{\pi}\left(\operatorname{Fix}\left(f_{\pi}\right)_{x^{\prime}}\right] .\right.
$$

Thus $\left[\operatorname{Fix}\left(\bar{f}_{\pi}\right)_{b}: p_{\pi}\left(\operatorname{Fix}\left(f_{\pi}\right)_{x}\right)\right]$ is a constant, denoted by $m$.

Then, from Corollary 5.4, for any $b \in \overline{\boldsymbol{F}_{\boldsymbol{i}}}, i=1, \cdots, n$, the length of each essential orbit of Fix $\left(\bar{f}_{\pi}\right)_{b}$ is equal to $m$. Hence $N_{K}\left(f_{b}\right)=$ $m \cdot C_{i}$ and $m$ is a factor of $N_{K}\left(f_{b}\right)$. Then (6) implies

$$
m \cdot N(f)=N(\bar{f}) \cdot N_{K}\left(f_{b}\right) .
$$

Since $N(f) \neq 0$, comparing this equation with (9), we get $P_{K}(f)=$ $m$.

For a space $X$, a map $f: X \rightarrow X$ and a point $x_{0} \in X$, let $J\left(f, x_{0}\right)$ denote the Jiang subgroup of $\pi_{1}\left(X, f\left(x_{0}\right)\right)$ consisting of the traces of $x_{0}$ under cyclic homotopies $f \simeq f$. Let $J\left(X, x_{0}\right)$ denote $J\left(\mathrm{id}_{X}, x_{0}\right) \subset$ 
$\pi_{1}\left(X, x_{0}\right)$. The group $J\left(X, x_{0}\right)$ is contained in the center of $\pi_{1}\left(X, x_{0}\right)$, hence it is a normal subgroup, so we may omit the base point and talk about $J(X) \subset \pi_{1}(X)$. In general $J\left(f, x_{0}\right)$ is not necessarily a normal subgroup of $\pi_{1}\left(X, f\left(x_{0}\right)\right)$. However, given a normal subgroup $H \subset \pi_{1}(X)$, we shall use the statement $H \subset J(f)$ to express the condition that for some (hence all) $x_{0} \in X, H\left(f\left(x_{0}\right)\right)$ is contained in $J\left(f, x_{0}\right)$. For example, we always have $J(X) \subset J(f)$. (Cf. [3], p. 101.)

LEMma 5.10. Let $X$ be a compact connected ANR and $H$ be a normal subgroup of $\pi_{1}(X)$. Let $f: X \rightarrow X$ be a map such that $H \subset$ $J(f)$ and $f(H) \subset H$. Suppose $\boldsymbol{F}, \boldsymbol{F}^{\prime} \in \nabla(f)$ make $\operatorname{id}_{\nu}(\boldsymbol{F})=\mathrm{id}_{\nu}\left(\boldsymbol{F}^{\prime}\right)$ $\left(\mathrm{id}_{\nabla}: \nabla(f) \rightarrow \nabla_{H}(f)\right.$ is induced by id: $X \rightarrow X$, cf. Corollary 1.6). Then $i_{f}(\boldsymbol{F})=i_{f}\left(\boldsymbol{F}^{\prime}\right)$.

Proof. Let $(x, w)$ be a reference pair for $f$. We need only to prove the lemma by use of representations in $(x, w)$. Take $\langle a\rangle$, $\langle b\rangle \in \pi_{1}(X, x)$ such that $\langle a\rangle \in \boldsymbol{F},\langle b\rangle \in \boldsymbol{F}^{\prime}$. Since $\mathrm{id}_{\nu}(\boldsymbol{F})=\mathrm{id}_{\nu}\left(\boldsymbol{F}^{\prime}\right)$, then $\left[\langle a\rangle_{H}\right]=\left[\langle b\rangle_{H}\right]$, and we can find an element $\langle r\rangle$ of $\pi_{1}(X, x)$ such that

$$
\langle a\rangle_{H}=\langle\boldsymbol{r}\rangle_{H}\langle b\rangle_{H} \tilde{f}_{H}\left(\langle\boldsymbol{r}\rangle_{H}^{-1}\right)=\left\langle\boldsymbol{r} b w\left(f \circ \boldsymbol{r}^{-1}\right) w^{-1}\right\rangle_{H} .
$$

Let $b^{\prime}=r b w\left(f \circ r^{-1}\right) w^{-1}$, then $\left\langle b^{\prime}\right\rangle \in[\langle b\rangle]=\boldsymbol{F}^{\prime}$ and $\left\langle a^{-1} b^{\prime}\right\rangle \in H$. Since $H \subset J(f)$ by assumption, i.e., $H(f(x)) \subset J(f, x)$, so $\left\langle a^{-1} b^{\prime}\right\rangle \in H(x) \subset$ $w_{*}^{-1} J(f, x)$. Now $\boldsymbol{F}^{\prime}=\left[\left\langle b^{\prime}\right\rangle\right]=\left[\langle a\rangle\left\langle a^{-1} b^{\prime}\right\rangle\right]$ differs from $\boldsymbol{F}$ by an element of $w_{*}^{-1} J(f, x)$ so $i_{f}(\boldsymbol{F})=i_{f}\left(\boldsymbol{F}^{\prime}\right)$. (Cf. [6].)

THEOREM 5.11. Let $(E, p, B)$ be orientable and let $f: E \rightarrow E$ be a fiber map with $N(f) \neq 0$. Suppose $\pi_{1}(E)$ and $\pi_{1}\left(p^{-1}(b)\right)$ are abelian. Then $P(f)$ equals the order of the kernel of the following homomorphism induced by $i_{b}: p^{-1}(b) \rightarrow E$

$$
\left(i_{b}\right)_{\nabla}^{\prime}: \pi_{1}\left(p^{-1}(b)\right) / \operatorname{Im}\left(1-\left(f_{b}\right)_{\pi}\right) \longrightarrow \pi_{1}(E) / \operatorname{Im}\left(1-f_{\pi}\right) .
$$

Proof. Take $x \in \Phi(f), b=p(x)$, and use $\left(x, e_{x}\right)$ as reference pair for $f$, so that $\widetilde{f}_{\pi}=f_{\pi}$. Consider the diagram

$$
\begin{gathered}
\nabla\left(f_{b} ; x, e_{x}\right) \stackrel{\left(i_{b}\right)_{\nabla}^{\prime}}{\longrightarrow} \nabla\left(f ; x, e_{x}\right) \\
\searrow \nearrow_{i d_{\triangleright} \searrow} \nearrow_{\left(i_{b}\right)_{\nabla}} \\
\nabla_{K}\left(f_{b} ; x, e_{x}\right) .
\end{gathered}
$$

Since $\pi_{1}(E)$ is abelian, $\nabla\left(f ; x, e_{x}\right)=\pi_{1}(E, x) / \operatorname{Im}\left(1-f_{\pi}\right)$ is an abelian group. Similarly $\nabla\left(f_{b} ; x, e_{x}\right)=\pi_{1}\left(p^{-1}(b), x\right) / \operatorname{Im}\left(1-\left(f_{b}\right)_{\pi}\right)$ and $\nabla_{K}\left(f_{b} ; x, e_{x}\right)=$ $\left(\pi_{1}\left(p^{-1}(b), x\right) / K\right) / \operatorname{Im}\left(1-\left(f_{b}\right)_{K}\right)$ are also abelian groups. Then, $\left(i_{b}\right)_{V}^{\prime}$ is just the homomorphism specified in the theorem, and $\left(i_{b}\right)_{\nabla}$ and $\mathrm{id}_{\nabla}$ 
are also homomorphisms. By Lemma 1.4, $\left(i_{b}\right)_{\nabla}^{\prime}=\left(i_{b}\right)_{\nabla} \circ \mathrm{id}_{\nabla}$. But $\mathrm{id}_{\nabla}$ is surjective (Corollary 1.6), so we get

$$
\sharp \operatorname{Ker}\left(i_{b}\right)_{V}^{\prime}=\left(\# \operatorname{Ker}\left(i_{b}\right)_{\nabla}\right) \cdot\left(\# \operatorname{Ker}\left(\operatorname{id}_{\nabla}\right)\right) \text {. }
$$

According to Lemma 5.2, $\operatorname{Ker}\left(i_{b}\right)_{\nabla}$ is exactly an orbit of Fix $\left(\bar{f}_{\pi}\right)_{b}$. Then by Corollary 5.4 and Theorem 5.9, we have

$$
\# \operatorname{Ker}\left(i_{b}\right)_{\nabla}=P_{K}(f) \text {. }
$$

According to [5], Corollary 1 on p. 50, $K_{b} \subset J\left(p^{-1}(b)\right)$. But $J\left(p^{-1}(b)\right) \subset$ $J\left(f_{b}\right)$, so, by Lemma 5.10 , for any $\boldsymbol{F} \in \nabla_{K}\left(f_{b}\right)$, all elements of $\operatorname{id}_{\bar{V}}^{-1}(\boldsymbol{F})$ have the same index. It follows that $N\left(f_{b}\right)=N_{K}\left(f_{b}\right) \cdot\left(\# \operatorname{Ker}(\mathrm{id})_{\nabla}\right)$ (because (id) ${ }_{\nabla}$ is surjective). Then

$$
\begin{aligned}
\# \operatorname{Ker}\left(i_{b}\right)_{\Gamma}^{\prime} N(f) & =\left(\# \operatorname{Ker}(\mathrm{id})_{\nabla}\right) \cdot\left(\# \operatorname{Ker}\left(i_{b}\right)_{\nabla}\right) \cdot N(f) \\
& =\left(\# \operatorname{Ker}(\mathrm{id})_{\nabla}\right) P_{K}(f) N(f) \quad \text { (Theorem 5.9) } \\
& \left.=\left(\# \operatorname{Ker}(\mathrm{id})_{\nabla}\right) N(\bar{f}) N_{K}\left(f_{b}\right) \quad \text { by }(9)\right) \\
& =N(\bar{f}) \cdot N\left(f_{b}\right) .
\end{aligned}
$$

It follows that $\sharp \operatorname{Ker}\left(i_{b}\right)_{\nabla}^{\prime}=P(f)$ (because $N(f) \neq 0$ ).

Corollary 5.12 (Pak [9]). Let $(E, p, B)$ be orientable and $f: E \rightarrow E$ be a fiber map such that $N(f) \neq 0$. Suppose that $\pi_{1}(E)$ is abelian and the fiber satisfies the Jiang condition $J\left(p^{-1}(b)\right)=\pi_{1}\left(p^{-1}(b)\right)$. Then $P(f) N(f)=N(\bar{f}) N\left(f_{b}\right)$, where $P(f)$ is the order of the kernel of $\left(i_{b}\right)_{\Gamma}^{\prime}$.

REMARK. If we assume that $f$ and $f_{b}$ are both eventually commutative instead of that $\pi_{1}(E)$ and $\pi_{1}\left(p^{-1}(b)\right)$ are abelian, then Theorem 5.11 still holds. We can prove that the condition " $f_{b}$ is eventually commutative" is independent of the choice of $b$, that is, if for some $b \in \Phi(\bar{f})$ the condition is satisfied, then for any other $b \in \Phi(\bar{f})$ it is also satisfied.

\section{REFERENCES}

1. R. Brooks, R. Brown, J. Pak and D. Taylor, The Nielsen number of maps of tori, Proc. Amer. Math. Soc., 52 (1975), 398-400.

2. R. Brown, The Nielsen number of a fibre map, Annals of Math., 85 (1967), 483-493.

3. - The Lefschetz Fixed Point Theorem, Scott-Foresman, Chicago, 1971.

4. E. Fadell, Natural fiber splittings and Nielsen numbers, Houston J. Math., 2 (1976), 71-84.

5. D. H. Gottlieb, on fiber spaces and the evaluation map, Annals of Math., 87 (1968), $42-55$.

6. Boju Jiang, Estimation of the Nielsen numbers, II, Acta Sci. Natur. Univ. Pekin, (1979), 48-57.

7. T. H. Kiang, The Theory of Fixed Point Classes (in Chinese), Scientific Fress, 
Peking, 1979.

8. D. McCord, An estimate of the Nielsen number and an example concerning the Lefschetz fixed point theorem, Pacific J. Math., 66 (1976), 195-203.

9. J. Pak, On the Reidemeister numbers and Nielsen numbers of fiber-preserving maps, preprint.

10. E. Spanier, Algebraic Topology, McGraw-Hill, New York, 1966.

Received October 22, 1980.

BeIJING UNIVERSITY

BeiJing, China 



\section{PACIFIC JOURNAL OF MATHEMATICS}

\section{EDITORS}

DONALD BABBITT (Managing Editor)

University of California

Los Angeles, California 90024

Hugo RossI

University of Utah

Salt Lake City, UT 84112

C. C. MOORE and ARTHUR Agus

University of California

Berkeley, CA 94720

\section{J. DUGUNDJI}

Department of Mathematics University of Southern California Los Angeles, California 90007

R. FinN and J. MILGRAM Stanford University Stanford, California 94305

\section{ASSOCIATE EDITORS}
R. ARNES
E. F. BeCKENBACH
B. H. Neumann
F. WOLF
K. YosHIDA

\section{SUPPORTING INSTITUTIONS}

UNIVERSITY OF ARIZONA

UNIVERSITY OF BRITISH COLUMBIA

CALIFORNIA INSTITUTE OF TECHNOLOGY

UNIVERSITY OF CALIFORNIA

MONTANA STATE UNIVERSITY

UNIVERSITY OF NEVADA, RENO

NEW MEXICO STATE UNIVERSITY

OREGON STATE UNIVERSITY
UNIVERSITY OF OREGON UNIVERSITY OF SOUTHERN CALIFORNIA STANFORD UNIVERSITY UNIVERSITY OF HAWAII UNIVERSITY OF TOKYO UNIVERSITY OF UTAH WASHINGTON STATE UNIVERSITY UNIVERSITY OF WASHINGTON 


\section{Pacific Journal of Mathematics}

Vol. 100, No. $1 \quad$ September, 1982

Charalambos D. Aliprantis, Owen Sidney Burkinshaw and M. Duhoux,

Compactness properties of abstract kernel operators $\ldots \ldots \ldots \ldots \ldots \ldots 1$

Roger C. Alperin, Locally compact groups acting on trees .............23

Robert F. Brown, Real homology of Lie group homomorphisms ......... 33

Karen Chase, Maximal groups in sandwich semigroups of binary relations . . 43

W. Wistar (William) Comfort and T. Soundararajan, Pseudocompact

group topologies and totally dense subgroups $\ldots \ldots \ldots \ldots \ldots \ldots \ldots 61$

M. Ferri and C. Gagliardi, Crystallisation moves $\ldots \ldots \ldots \ldots \ldots \ldots \ldots$

Kenneth R. Goodearl, Directly finite aleph-nought-continuous regular

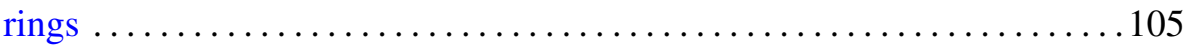

Edward Lewis Green, On the representation theory of rings in matrix

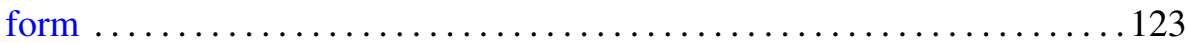

Walter Hengartner and Glenn E. Schober, Interpolation, continuation, and

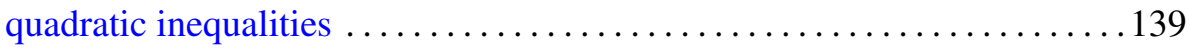

Kenneth Kunen and Haskell Paul Rosenthal, Martingale proofs of some geometrical results in Banach space theory $\ldots \ldots \ldots \ldots \ldots \ldots \ldots \ldots \ldots \ldots$

Brian William McEnnis, Shifts on indefinite inner product spaces. II . . . . . 177

Roman Pol, Note on the spaces $P(S)$ of regular probability measures whose topology is determined by countable subsets $\ldots \ldots \ldots \ldots \ldots \ldots \ldots \ldots 185$

Joan Manuel Verdera Melenchón, Finitely generated projective extensions

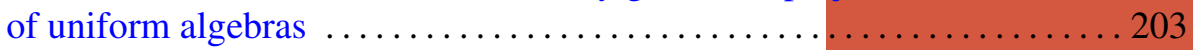

Cheng Ye You, Fixed point classes of a fiber map .................. 217 